\title{
Disruption of lineage specification in adult pulmonary mesenchymal progenitor cells promotes microvascular dysfunction
}

\author{
Christa F. Gaskill, ${ }^{1}$ Erica J. Carrier, ${ }^{1}$ Jonathan A. Kropski, ${ }^{1}$ Nathaniel C. Bloodworth, ${ }^{2}$ Swapna Menon, ${ }^{3}$ Robert F. Foronjy, ${ }^{4}$ \\ M. Mark Taketo, ${ }^{5}$ Charles C. Hong, ${ }^{1,6}$ Eric D. Austin, ${ }^{7}$ James D. West, ${ }^{1}$ Anna L. Means, ${ }^{8}$ James E. Loyd, ${ }^{1}$ W. David Merryman, ${ }^{2}$ \\ Anna R. Hemnes, ${ }^{1}$ Stijn De Langhe, ${ }^{9}$ Timothy S. Blackwell, ${ }^{1}$ Dwight J. Klemm, ${ }^{10,11}$ and Susan M. Majka ${ }^{1,12}$

\begin{abstract}
'Department of Medicine, Division of Allergy, Pulmonary and Critical Care Medicine or Division of Cardiovascular Medicine, Vanderbilt University Medical Center, Nashville, Tennessee USA. ${ }^{2}$ Department of Biomedical Engineering, Vanderbilt University, Nashville, Tennessee USA. ${ }^{3}$ Pulmonary Vascular Research Institute, Kochi, and AnalyzeDat Consulting Services, Kerala, India. ${ }^{4}$ Department of Medicine, Division of Pulmonary and Critical Care Medicine, SUNY Downstate Medical Center, Brooklyn, New York, USA. ${ }^{5}$ Kyoto University, Sakyo, Kyoto, Japan. ${ }^{6}$ Department of Pathology and Laboratory Medicine or Department of Medicine, Veterans Affairs Tennessee Valley Healthcare System, Nashville, Tennessee, USA. ${ }^{7}$ Department of Pediatrics, and ${ }^{8}$ Department of Surgery, Vanderbilt University Medical Center, Nashville, Tennessee, USA. ${ }^{9}$ National Jewish Health, Denver, Colorado, USA. ${ }^{10}$ Department of Medicine, Pulmonary and Critical Care Medicine, Gates Center for Regenerative Medicine and Stem Cell Biology, University of Colorado, Aurora, Colorado, USA. "'Ceriatric Research Education and Clinical Center, Eastern Colorado Health Care System, Denver, Colorado, USA. ${ }^{12}$ Vanderbilt Center for Stem Cell Biology,
\end{abstract} \\ Vanderbilt University, Nashville, Tennessee, USA.
}

\begin{abstract}
Pulmonary vascular disease is characterized by remodeling and loss of microvessels and is typically attributed to pathological responses in vascular endothelium or abnormal smooth muscle cell phenotypes. We have challenged this understanding by defining an adult pulmonary mesenchymal progenitor cell (MPC) that regulates both microvascular function and angiogenesis. The current understanding of adult MPCs and their roles in homeostasis versus disease has been limited by a lack of genetic markers with which to lineage label multipotent mesenchyme and trace the differentiation of these MPCs into vascular lineages. Here, we have shown that lineage-labeled lung MPCs expressing the ATP-binding cassette protein ABCC2 $\left(\mathrm{ABCC2}^{+}\right)$are pericyte progenitors that participate in microvascular homeostasis as well as adaptive angiogenesis. Activation of Wnt/ $\beta$-catenin signaling, either autonomously or downstream of decreased BMP receptor signaling, enhanced ABCC2+ MPC proliferation but suppressed MPC differentiation into a functional pericyte lineage. Thus, enhanced Wnt/ $\beta$-catenin signaling in ABCG2+ MPCs drives a phenotype of persistent microvascular dysfunction, abnormal angiogenesis, and subsequent exacerbation of bleomycin-induced fibrosis. ABCC2 ${ }^{+}$MPCs may, therefore, account in part for the aberrant microvessel function and remodeling that are associated with chronic lung diseases.
\end{abstract}

\section{Introduction}

Microvascular dysfunction is a key component of chronic lung disease, which is the fourth-leading cause of mortality worldwide. Changes in distal lung tissue structure include enlarged, obstructed, or fibrotic airspaces, decreased microvascular density, and ultimately loss of gas exchange surfaces. A common comorbidity in chronic lung diseases is vasculopathy, or pulmonary vascular dysfunction (PVD), characterized by remodeling and loss of microvessels, which substantially worsens prognosis and limits survival. The relevance of neovascularization to the pathophysiology of PVD has not been resolved, as conflicting evidence depicts angiogenesis as both reparative and pathologic.

The importance of identifying barriers to vascular regeneration in the adult lung is underscored by the current emphasis on cell therapy and bioengineering approaches to address pulmonary vascular pathologies. However, without understanding the endog-

Conflict of Interest: The authors have declared that no conflict of interest exists. Submitted: May 18, 2016; Accepted: March 2, 2017.

Reference information: / Clin Invest. 2017;127(6):2262-2276.

https://doi.org/10.1172/JCl88629. enous cells that mitigate damage and promote healing, targeted cell or small-molecule therapies and 3D tissue reconstruction will not achieve success. Adult tissue regeneration and repair may in part be attributed to tissue-resident stem cells within the mesenchymal compartment that are capable of vascular differentiation (1). To date, our understanding of the basic biology of adult pulmonary MPCs and the roles they play in microvascular homeostasis versus disease is limited.

MPCs have long been thought to be the "mural" precursor to a differentiated pericyte $(2,3)$. Interestingly, pericytes have also been hypothesized to be mesenchymal stem cells in adult tissue (4), however, recent studies using lineage tracing have disproved this theory (5). The current understanding of the role pericytes and their mesenchymal precursors play in adult lung disease, de novo angiogenesis, and vascular remodeling is controversial, as conflicting evidence depicts angiogenesis as both reparative and pathologic (6-8). The consensus is that pericytes differentiate toward a myofibroblast lineage and participate in pathological processes. However, a specific role for these cells during fibrosis or adaptive angiogenesis has not yet been defined (9-16). While these hypotheses are intriguing, the studies of tissue-resident 
stromal progenitor cells as well as the origin of pericyte lineages in the adult have been complicated by a lack of unique markers to define specific cell types within heterogeneous mesenchymal pericyte precursors and lineage-specified pericyte populations (Supplemental Table 1; supplemental material available online with this article; https://doi.org/10.1172/JCI88629DS1). A second limitation is the ability to translate findings from cell populations identified in murine tissues to human cells.

In accordance with these gaps in knowledge, the cell-specific mechanisms that regulate the development and progression of the microvascular dysfunction associated with PVD in chronic adult lung diseases are not well defined. PVD is often asymptomatic until severe, when approximately $50 \%$ to $70 \%$ of the pulmonary vascular bed is lost, both in structure and function, at which point pulmonary vascular resistance and pulmonary artery pressure rise, leading to a diagnosis of pulmonary hypertension $(\mathrm{PH})$ or pulmonary artery hypertension (PAH) (17). Major risks for the development of PVD and PH include genetic mutation, environment (hypoxia, drugs), and immune deregulation and associated diseases $(17,18)$. To date, the etiology of microvascular remodeling underlying PVD remains unknown and is complicated by a lack of rodent models recapitulating early-stage vasculopathy in the absence of or prior to injury. The current limited understanding of microvascular dysfunction as a precursor to PVD and the lack of diagnostic approaches and criteria emphasize the necessity to understand the mechanisms of microvascular dysfunction in both rodent models and the clinical setting.

To address the aforementioned issues, our laboratory has identified a unique and traceable subpopulation of perivascular, multipotent, lineage-negative MPCs in both adult mouse and human lung on the basis of their expression of ATP-binding cassette subfamily $\mathrm{G}$ member 2 (ABCG2). Using a combination of flow cytometry and a low-dose tamoxifen induction lineagetracing strategy, we defined ABCG2 as a marker to reproducibly label a subpopulation of adult lung MPCs $(12,19)$. In these studies, we demonstrate for the first time to our knowledge that ABCG2 lung MPCs participate in microvascular homeostasis and distal lung tissue structure via pericyte specification and differentiation. We show, in both murine and human ABCG2 $2^{+}$lung MPCs, that increased $\mathrm{Wnt} / \beta$-catenin signaling results in abnormal expansion as well as pericyte specification of MPCs without normal pericyte maturation, driving a phenotype of persistent microvascular dysfunction. In response to genetic depletion of BMP receptor type II (BMPR2), stabilization of $\beta$-catenin, or bleomycin induced fibrosis, the resultant MPC-derived pericytes resemble myofibroblasts. However, the deregulated pericytes participate in adaptive angiogenesis and exacerbate fibrosis as opposed to directly contributing to fibrotic scar formation. Defects in $\mathrm{ABCG}^{+}$pericyte progenitor maturation elicit pathological changes in the microvasculature that mirror vasculopathy before overt clinical symptoms of disease appear. This suggests that a better understanding of the role of these MPCs in the maintenance of microvascular integrity in the lungs may lead to earlier diagnosis and treatment of PVD.

\section{Results}

Phenotypic similarities between conditional ABCG2-restricted depletion of BMPR 2 and stabilization of $\beta$-catenin: alteration of microvas- cular homeostasis in the adult lung. Reactivation of the developmental signaling pathways is often associated with abnormal lineage specification and disease pathology in adult tissues. Coordinated Wnt and BMPR2 signaling influence the proliferation and survival of endothelium and smooth muscle during angiogenesis as well as vascular remodeling of the pulmonary circulation (20-22). We therefore sought to determine how their coordinated signaling in resident lung $\mathrm{ABCG} 2^{+}$mesenchymal precursors regulates pulmonary microvascular homeostasis in vivo and in vitro. To test the hypothesis that resident lung $\mathrm{ABCG} 2^{+} \mathrm{MPCs}$ contribute to microvascular homeostasis and that disruption of Wnt/BMPR2 signaling in MPCs leads to the development of microvascular dysfunction in vivo, we exploited the inducible ABCG2 $2^{+}$lineage-labeling strategy (Figure 1A). Wnt signaling was enhanced in ABCG2 $2^{+}$lung MPCs in vivo by induced expression of a mutant $\beta$-catenin. This model was achieved by crossing a lox-stop-lox-mutant $\beta$-catenin exon 3 mouse (Ctnnb1 $1^{\text {LSL-ex3 }}$ mouse), which removes glycogen synthase kinase $3 \beta$ (GSK3 $\beta$ ) phosphorylation sites that target $\beta$-catenin for degradation, with bigenic lineage-labeling Abcg2-CreERT2 Rosa26 $m T \operatorname{Tm} G$ mice (23-25). Adult trigenic mice were also engineered to genetically deplete 1 copy of Bmpr 2 by crossing Abcg2$\mathrm{Cre}^{E R T 2}$, Rosa26-mTmG (23), and Bmpr $2^{f / f l}$ (26) lines, resulting in Abcg2-Cre ${ }^{E R T 2} \mathrm{Bmpr} 2^{f l / f l}$ Rosa26-mTmG mice (referred to herein as $B m p r 2^{f l /+}$ mice). In these optimized low-dose tamoxifen systems, $\mathrm{ABCG}^{+}$lung MPCs expressed enhanced GFP (eGFP) and 1 copy of BMPR 2 or stabilized $\beta$-catenin (Figure 1B).

Genetic depletion of BMPR2 or stabilization of $\beta$-catenin in $\mathrm{ABCG}^{+}$lung MPCs resulted in their expansion (Figure 1, B and $\mathrm{D}-\mathrm{F})$. Increased numbers of eGFP-expressing ABCG2+ lung MPCs were present on days 2 and 14 after induction in complementary flow cytometric and immunostaining analyses (Figure 1, B and D-F, and Supplemental Figure 1A). The increased fluorescence staining intensity observed in the tissue sections versus the data shown in the flow cytometric dot plots is likely due to the detection of multiple long cell processes emanating from a single MPC (Figure 1, D-F). Dysfunctional BMPR2 and Wnt signaling in $\mathrm{ABCG}^{+}$lung MPCs also increased the microvessel dysfunction documented by vascular leak on day 14 (Figure 1C). The expanded pool of ABCG2 $2^{+}$lung MPCs in both $B m \mathrm{pr}^{\mathrm{fl}+}$ and $\beta$-catenin-overexpressing ( $\beta \mathrm{OE}$ ) mice was retained after 18 to 20 weeks in vivo (Supplemental Figure 2A). Proliferation of ABCG2+ lung MPCs was detected by proliferating cell nuclear antigen (PCNA) and eGFP localization on days 2 and 14 in lung tissue sections (Supplemental Figure $1 \mathrm{C}$ and Supplemental Figure 3, A-C). The $\mathrm{ABCG}^{+}$lung MPCs did not colocalize with large arterioles or smooth muscle $\alpha$ actin-expressing ( $\alpha$-SMA-expressing) cells (Figure 1, L-T, and Supplemental Figure 1B).

To evaluate the role of Wnt and BMPR2 signaling in $\mathrm{ABCG} 2^{+}$ lung MPCs in lung microvascular homeostasis, we analyzed WT, $B m p r 2^{f l /+}$, and $\beta O E$ mice at 20 weeks (Figure $1 \mathrm{~A}$ and Supplemental Figure 4A). Lung microvascular function and structure were assessed by right ventricular systolic pressure (RVSP) as well as morphometric measurements. We found that both Bmpr $2^{f l+}$ and $\beta O E$ mice had a subtle increase in RVSP, suggesting a microvascular defect (Figure 1G). Morphometric analysis identified a corresponding loss of microvessel density in $B m p r 2^{f /+}$ mice that was more variable in the $\beta \mathrm{OE}$ strain (Figure $1, \mathrm{H}-\mathrm{J}$ ) in the absence of 

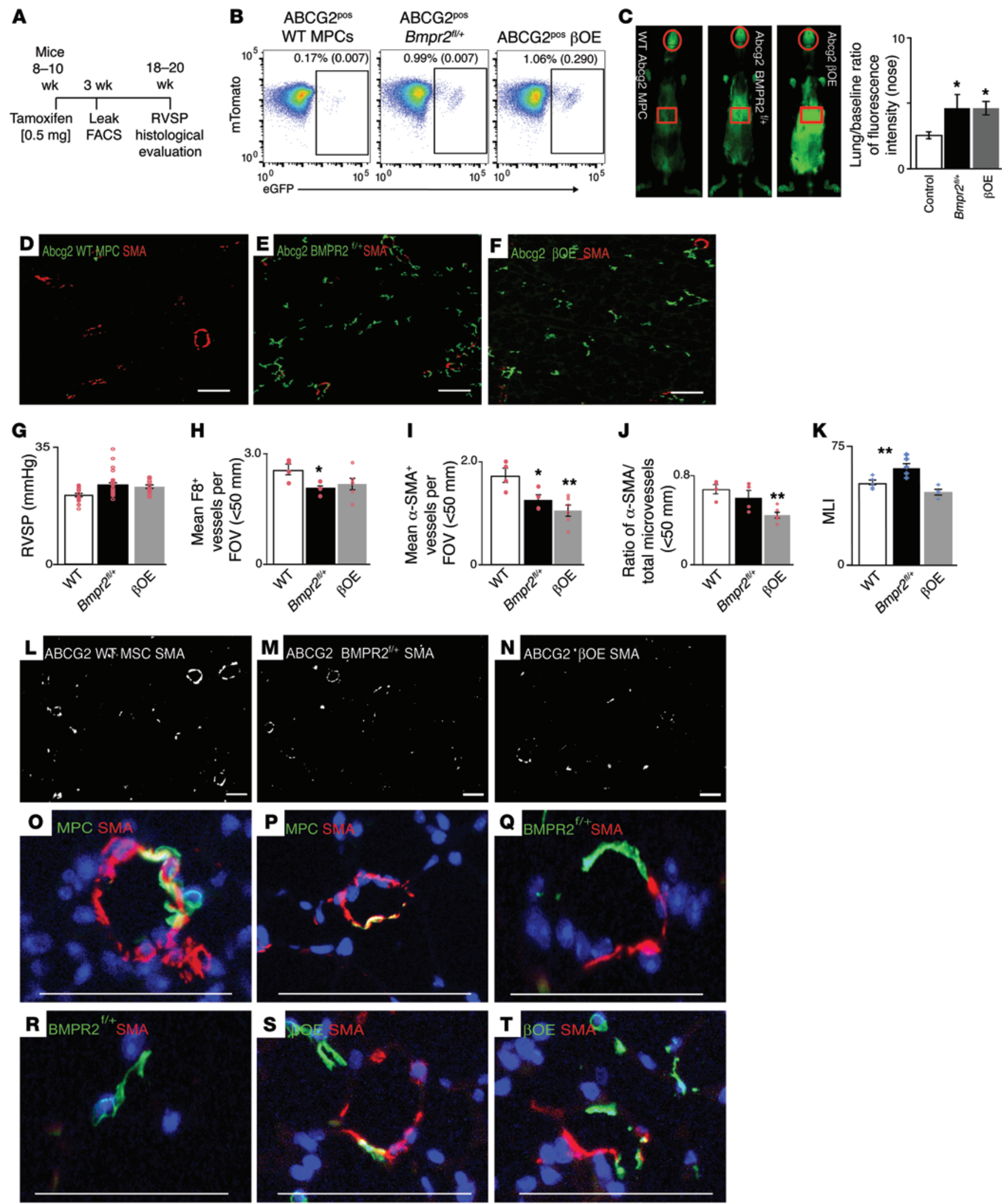
Figure 1. Abnormal pericyte lineage specification and microvascular dysfunction accompanied by loss of distal lung tissue structure in vivo via deregulation of BMPR2 and Wnt/ $\beta$-catenin signaling in lung MPCs. (A) Schematic time line of mouse modeling. WT, $B \mathrm{mpr} 2^{\mathrm{fl} /+}$, and $\beta O E$ mice were induced with i.p. tamoxifen ( $0.5 \mathrm{mg}$ total). (B) Two weeks after induction, eGFP labeling and enumeration of ABCG2+ lung MPCs were confirmed by flow cytometry $(n=3-6)$. Numbers in parentheses represent SEM. (C) Three weeks after induction, pulmonary vascular leak was quantitated. Representative images of mice obtained with the Pearl analyzer. Bar graph shows the ratio calculated for each animal, comparing the intensity of dye in the lung (red squares) with the baseline fluorescence in the nose (red circles) $(n=7-15)$. (D-F) Additional mice were sacrificed and the lungs agarose inflated using constant pressure to obtain lung tissue for immunofluorescence analyses. Immunostaining for detection of eGFP (green) MPCs and derivatives as well as $\alpha$-SMA (red) was performed $(n=4)$. (C) Eighteen to twenty weeks after tamoxifen induction, RVSP was measured ( $n=$ 10-13). Immunostaining was performed on lung tissue sections to detect (H) F8+ microvessels and (I) $\alpha-5 M A^{+}$muscularized vessels. (J) The ratio of $\alpha$-SMA/total microvessels was also calculated, and the positive numbers were counted per FOV. Six to eight sections of twenty FOV per section were evaluated $(n=4)$. (K) MLI was calculated to evaluate the loss of distal lung tissue structure $(n=6)$. ( $\mathbf{L}-\mathbf{N})$ Immunofluorescent staining (IFC) to detect $\alpha$-SMA demonstrated a decrease in $\alpha$-SMA overall in $B m p r 2^{f / /+}$ and $\beta O E$ lungs. (L-T) Immunostaining was performed to detect ABCG2+ MPCs and derived eGFP- and $\alpha$-SMA-expressing cells. Scale bars: $50 \mu \mathrm{m}(\mathbf{L}-\mathbf{T})$ and $100 \mu \mathrm{m}(\mathbf{D}-\mathbf{F})$. Data are presented as the mean \pm SEM. ${ }^{*} P<0.05$ and ${ }^{*} P<0.01$, by 1-way ANOVA followed by Tukey's honest significant difference (HSD) post-hoc test.

increased muscularization of microvessels, collagen deposition, and inflammatory infiltrate (Figure $1 \mathrm{~K}$, and Supplemental Figure 2, B and C). Strikingly, both the $B m p r 2^{f /+}$ and $\beta O E$ mice had significantly decreased numbers of $\alpha$-SMA ${ }^{+}$microvessels (Figure 1, $\mathrm{D}-\mathrm{F}, \mathrm{I}$, and $\mathrm{L}-\mathrm{N}$ ), suggesting a decrease in differentiated pericytes. We also performed analyses to detect colocalization of eGFPderived MPCs and coagulation factor 8 (F8) or $\alpha$-SMA. No colocalization of MPC-derived cells or vascular markers was detected. The decreased microvessel density in the $B m p r 2^{f /+}$ mice paralleled a loss of distal lung tissue structure or airspace enlargement, as measured by the increased mean linear intercept (MLI) (Figure $1 \mathrm{~K})$. Eighteen to twenty weeks after induction, lineage-tracing analyses of $\mathrm{ABCG}^{+}$lung MPCs from $B m p r 2^{f / /+}$ and $\beta \mathrm{OE}$ mice demonstrated that perivascular eGFP-expressing MPCs or derived cells did not colocalize with $\alpha$-SMA-expressing cells (Figure $1, \mathrm{Q}-\mathrm{T}$ ), whereas the WT MPCs did colocalize with $\alpha$-SMA (Figure 1, O and P). The lack of $\alpha$-SMA expression by microvascular pericytes as a result of BMPR2 inactivation and $\beta$-catenin stabilization suggests that defects in function and differentiation of the expanded MPC pool may underlie the observed decrease in microvessel density and loss of tissue structure. Taken together, these data illustrate that microvessel loss adversely affects distal lung tissue structure and that the $B m p r 2^{f /+}$-driven phenotype is only partially dependent on increased $\beta$-catenin signaling.

Conditional stabilization of $\beta$-catenin or depletion of BMPR 2 in murine ABCG2 MPCs parallels a loss of clonogenic potential and increased pericyte lineage specification without maturation. To confirm that BMPR2 and Wnt/ $\beta$-catenin signaling in $A B C G 2^{+}$lung MPCs regulates their stem cell characteristics, we performed CFU-fibroblast (CFU-F) analyses to enumerate the presence of clonogenic progenitors and flow cytometric analyses to charac- terize the cell-surface determinants characteristic of murine and human MPCs. We also analyzed the expression of pericyte lineage specification via protein and gene expression. Isolation and characterization of murine $\mathrm{ABCG} 2^{+}$lung MPCs paralleled our findings in the in vivo models and in human MPC lines. Both $B m p r 2^{f / t}$ and $\beta O E \mathrm{ABCG}^{+}$murine lung MPCs showed a decreased ability to form CFU-F, which is indicative of a loss of functional clonogenic progenitors (Supplemental Figure 4B) (27). We observed that the loss of clonogenic potential paralleled an increase in pericyte lineage specification compared with WT MPCs, keeping in mind that lineage specification is not equal to terminal differentiation into mature and functional contractile pericytes $(28,29)$.

$B m p r 2^{f /+}$ and $\beta O E A B C G 2^{+}$lung MPCs also had significantly increased gene and protein expression of the pericyte markers chondroitin sulfate proteoglycan 4 (Cspg4, encoding neural/glial antigen 2 [NG2]) and regulator of $\mathrm{G}$ protein signaling 5 (Rgs5) (Figure 2, A and B), similar to that observed with BMPR2 inhibition in WT ABCG2+ MPCs (30). In the WT MPCs, we detected NG2 as a single band, while multiple bands were present at varying intensities in the $\beta \mathrm{OE}$ and Bmpr $2^{f / /+}$ MPCs (Figure 2B). These multiple bands are the result of extracellular processing of the NG2 proteoglycan that may then regulate signaling as well as migration (31). We again identified increased gene and protein expression of the Wnt $/ \beta$-catenin target WNT1-inducible signaling pathway protein 1 (Wisp1) (Figure 2, A and B) in Bmpr2 $2^{f /+}$ MPCs, which may regulate mesenchymal proliferation (32). We also observed altered gene expression of Wnt inhibitors, with a decrease in secreted frizzled-related protein 2 (Sfrp2) expression, while $S f r p 1$ expression increased in the $\beta O E$ ABCG2 $2^{+}$MPC line. Bmpr $2^{f /+}$ and $\beta O E$ $\mathrm{ABCG} 2^{+}$MPCs showed increased expression of CD146 (Figure 2C and Supplemental Figure $4 \mathrm{H}$ ), which is associated with pericyte or endothelial lineage specification from MPCs $(33,34)$. $\beta O E$ MPCs also had decreased expression of CD105 (also known as endoglin), indicative of an attenuated potential for terminal myogenic or $\alpha$-SMC differentiation and increased invasiveness $(35,36)$. To characterize $\beta$-catenin and its target, cyclin D1, we performed immunostaining on MPCs. $\beta$-Catenin localized to the membrane tight junctions or cytoplasm in WT cells, while cyclin D1 was not detected (Figure 2D). In contrast, we found that $\mathrm{Bmpr} 2^{\mathrm{fl} / \mathrm{t}}$ and $\beta \mathrm{OE}$ ABCG2+ $2^{+}$PCs demonstrated decreased $\beta$-catenin localization to the membrane tight junctions. Furthermore, cyclin D1 was highly immune reactive in the cytoplasm of $B m p r 2^{f / /}$ MPCs and in the nuclei of $\beta O E$ MPCs. Cytoplasmic cyclin D1 can regulate invasion as well as survival (37), while nuclear localization is typically associated with proliferation and survival (38).

Second, we documented that suppression of the BMPR signaling pathway increased Wnt $/ \beta$-catenin activity as well as expression of Cspg4 (NG2) and Wnt targets in isolated murine WT ABCG2 ${ }^{+}$MPCs using the small-molecule BMPR signaling inhibitor 4-[6-[4-(1-methylethoxy)phenyl]pyrazolo[1,5-a]pyrimidin-3-yl]-quinoline (DMH) (Figure 2E and Supplemental Figure $5, \mathrm{~A}$ and B). DMH inhibits SMAD1/5/8 phosphorylation in a BMPR1-specific manner, inhibiting signaling of both BMPR1 and BMPR2 (39). Activation of Wnt signaling correlated with increased gene expression of the pericyte marker Cspg4 (NG2) (28), decreased gene expression of Wnt inhibitors (dickkopf Wnt signaling pathway inhibitor $1[D k k 1]$ and $S f r p 1 / 2)$ and collagen 1 (Col1a1), as well as 
A
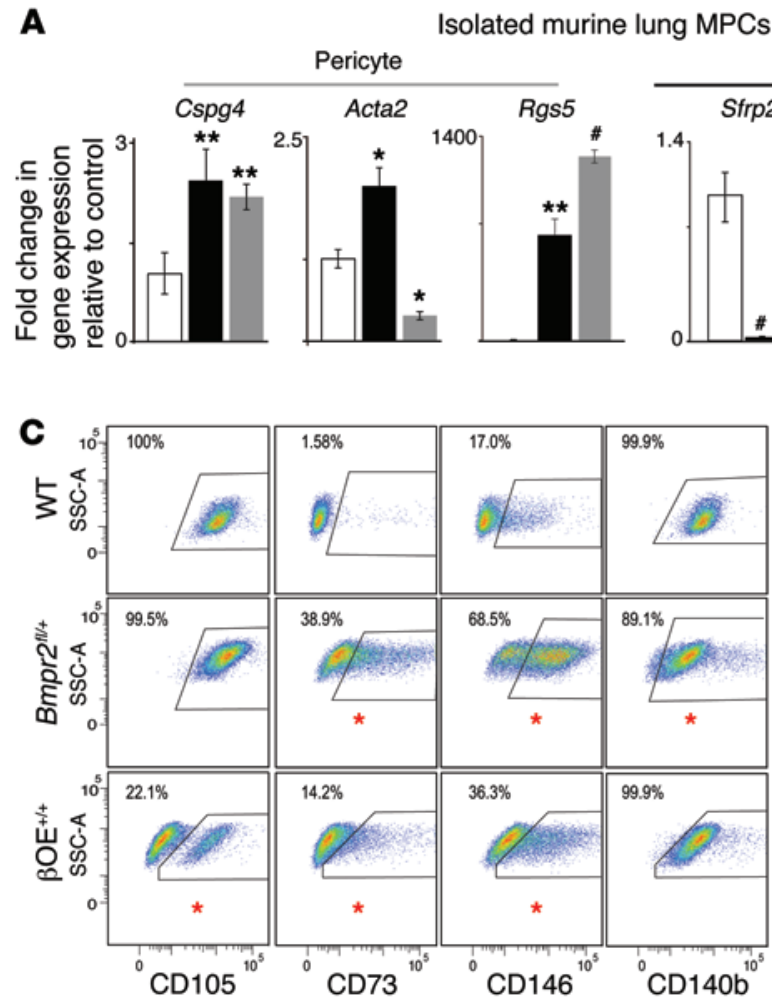

Wnt/ $\beta$-catenin

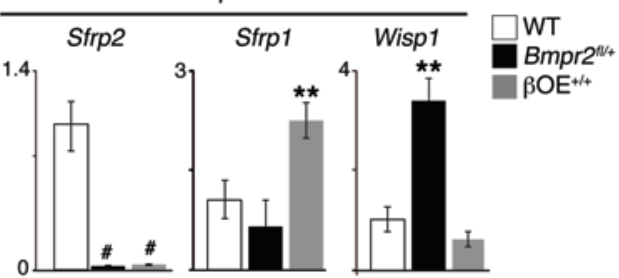

B

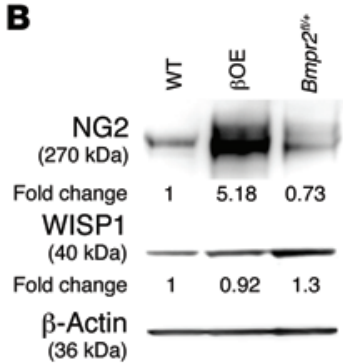

D
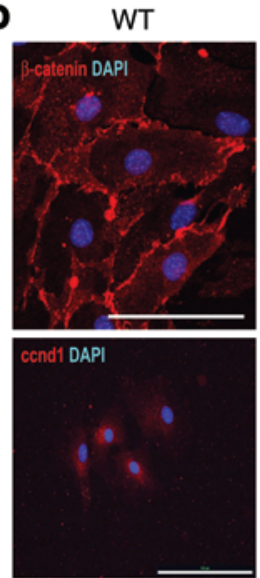

Bmpr2 $2^{f /+}$
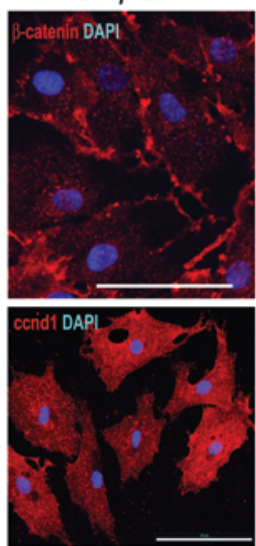

$\beta O E$

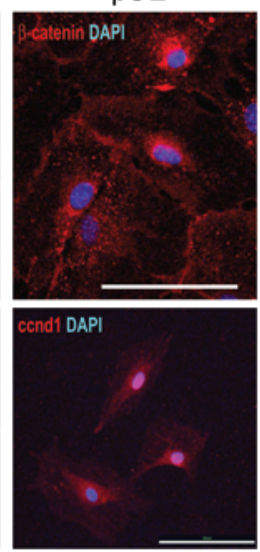

$\square$ Vehicle control DMH1

E

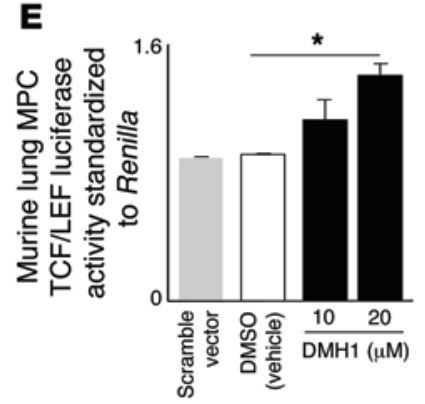

F Pericytes

Wnt/ $\beta$-catenin
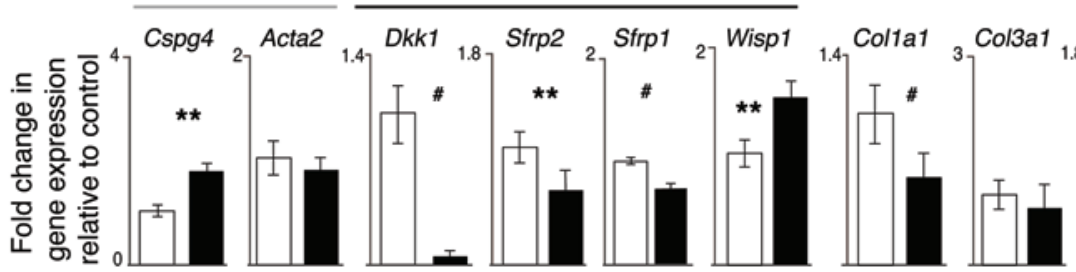

Fn ${ }_{4}^{4}$ Cond 1

G

Human WT vs. PAH lung MPCs
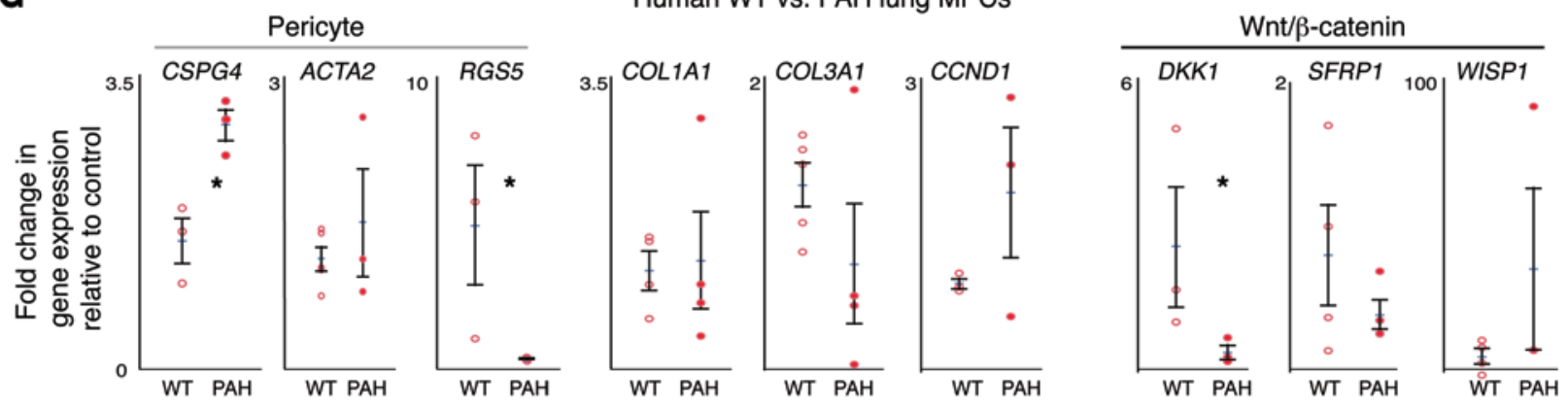

H Human lung MPCs Control HPAH IPAH

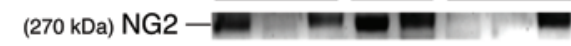

(40 kDa) WISP1 - - - - -

(36 kDa) Cyclin D1 -

$160 \mathrm{nea6}$

(36 kDa) $\beta$-Actin -

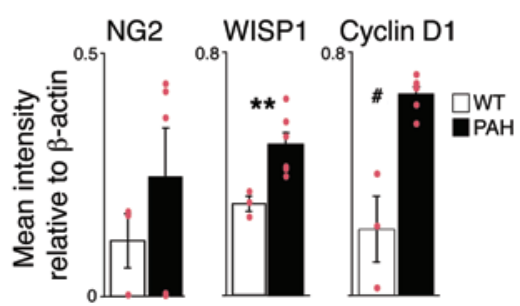


Figure 2. Isolated murine and human ABCC2+ lung MPCs demonstrate increased pericyte lineage specification in response to decreased BMPR2 or increased Wnt/B-catenin signaling. (A-F) BMPR2 knockdown and canonical Wnt activation in murine ABCG2+ lung MPCs increased pericyte lineage commitment. Sixteen weeks after induction, WT, Bmpr $2^{f / /+}$, and $\beta O E$ MPCs from lineage-labeled mice were isolated by flow sorting to detect eGFP fluorescence. (A) Following expansion, qRT-PCR analysis was performed to examine the expression levels of pericyte lineage-specific genes and canonical Wnt targets. (B) Western blot analysis was performed to quantitate the expression of NG2 and WISP1 proteins. A representative blot from two independent blots presented. (C) Isolated MPCs were stained with antibodies to detect cell-surface determinants characteristic of MPCs and pericytes. Gates were set based on fluorescence minus one control (FMO) controls. SSC-A, side scatter, area scaling. (D) Immunostaining was performed to localize $\beta$-catenin or cyclin D1 (Ccnd1) (red) in isolated cells (blue $=$ DAPI-stained nuclei). Scale bars: $100 \mu \mathrm{m}$. (E and F) BMP signaling was decreased in isolated murine WT lung MPCs using the small-molecule inhibitor DMH1, independently 2 times. (E) Canonical Wnt signaling activity was measured 48 hours after transfection using a TCF/ LEF dual-luciferase reporter assay and repeated independently 3 times. (F) Canonical Wnt target and pericyte lineage gene expression levels were evaluated after 48 hours by qRT-PCR. (G and $\mathbf{H}$ ) Human PAH ABCG2+ lung MPCs. (G) qRT-PCR analyses of control, HPAH, and IPAH human ABCG2+ lung MPCs were performed to quantitate the relative levels of gene expression for the pericyte lineage markers CSPG4, ACTA2, RCS5, the Wnt pathway targets DKK1, SFRP1, and WISP1, as well as the matrix proteins COL1A1 and COL1A3 and the cell-cycle regulator CCND1 $(n=3-4)$. (H) Protein levels of NG2 and the $\beta$-catenin target cyclin D1 were quantified by Western blotting $(n=3-5)$. Each assay was performed independently 2 times. Data are presented as the mean \pm SEM. ${ }^{*} P<0.05$, ${ }^{* *} P<0.01$, and $\# P<0.001$, by 1-way ANOVA followed by Tukey's post-hoc test (for murine qRT-PCR) and a nonparametric Wilcoxon-Kruskal-Wallis test and $\chi^{2}$ approximation (for patients' samples).

increased expression of $\beta$-catenin targets (fibronectin, cydlin D1 [Ccnd1], and Wisp1) (Figure 2F). Increased expression of WISP1 protein was also confirmed by Western blot analysis (Supplemental Figure 4E). Therefore, in ABCG2+ lung MPCs, suppression of BMPR2 signaling activates Wnt/ $\beta$-catenin signaling in vitro, which correlates with pericyte lineage specification.

Characterization of the human $\mathrm{ABCG} 2^{+}$lung MPCs paralleled our findings in the murine models, in that the PAH patients' MPC samples with abnormal BMPR2 signaling (Supplemental Figure 6C) showed decreased CFU-F potential (Supplemental Figure $6 \mathrm{E}$ ), while expressing characteristic MPC cell-surface determinants (Supplemental Table 2) $(27,40)$. Human PAH ABCG2+ lung MPCs also showed increased gene expression of the pericyte marker CSPG4 (28) and the Wnt target and regulator of proliferation CCND1 (Figure 2G). We found that collagen expression was not significantly changed, reminiscent of the murine in vivo model. We detected decreased gene expression for the Wnt inhibitor DKK1 and the regulator of $G$ protein signaling $R G S 5$. RGS5 is expressed by pericytes and correlates with maturation, contraction, and vasoreactivity $(41,42)$ as well as activation, an angiogenic switch favoring neovascularization and vessel remodeling in development, repair, and pathology (43). Gene expression was paralleled by increased protein expression of the pericyte marker NG2 and the Wnt targets WISP1 and cyclin D1 (Figure 2H). WISP1 positively regulates BMP signaling by enhancing BMP2 ligand activity and is also upregulated in pulmonary fibrosis $(32,44)$. These changes in gene expression were MPC specific, as they were not identifiable in lung fibro- blasts from patients with PH (Supplemental Figure 6F). Increased expression of the regulators of mesenchymal proliferation WISP1 and CCND1 and deregulated pericyte differentiation highlight $\mathrm{ABCG} 2^{+} \mathrm{MPCs}$ as candidates for the proliferative and undifferentiated cells that comprise vascular lesions and that are characteristic of human pathology and rodent models of disease $(45,46)$. ACTA2 (encoding $\alpha$-SMA) levels were variable among patients' samples, and overall, the levels were not significantly different from those detected in WT samples, suggesting that the underlying mechanisms of decreased contraction may vary by cell-specific defect and involve deregulated nucleation of actin filaments, stress fiber formation, as well as defective terminal differentiation (47).

Abnormal ABCG2 $2^{+}$MPC pericyte lineage maturation decreases microvascular contractility both in vivo and in vitro. A unifying feature of our ABCG2-driven murine models of microvascular dysfunction is the identification of decreased $\alpha$-SMA-invested microvasculature, complemented in vitro by pericyte specification in the absence of full maturation. Together, our findings highlight a defect in the maturation of pericytes from immature to mature contractile pericytes. These finding are reminiscent of the plasticity described for vascular smooth muscle cells (vSMCs), which are characterized by 2 major phenotypes: differentiated and contractile and dedifferentiated and synthetic (48-50). Therefore, to evaluate whether the loss of $\alpha$-SMA expression translates into a functional vascular defect, we quantified vascular contractility in vivo and in isolated MPCs in vitro. We measured the contractility of pulmonary vessels indirectly via RVSP measurement in ABCG2+ lung MPCs in Bmpr2$\mathrm{KO}$ and $\beta \mathrm{OE}$ models in response to acute epinephrine challenge. Both mouse models showed decreased contraction relative to that seen in WT mice, as indicated by the significantly decreased RVSP (Figure 3, A and B). We then analyzed isolated murine and human $\mathrm{ABCG} 2^{+} \mathrm{MPCs}$ in complementary in vitro contraction assays. $\mathrm{ABCG} 2^{+}$lung MPCs derived from $B m p r 2^{f /+}$ and $\beta O E$ mouse lungs as well as from patients with heritable PAH (HPAH) and patients with idiopathic PAH (IPAH) demonstrated significantly less contractility than did WT control MPCs (Figure 3C).

Increased $\beta$-catenin signaling is associated with abnormal differentiation of $A B C G 2^{+}$lung MPCs and exacerbation of bleomycininduced fibrosis in vivo. Mesenchymal cell differentiation into pericytes and myofibroblasts was proposed, beginning in 1970, as being associated with both wound healing and scar formation (2), as well as with fibrotic remodeling in the lung, kidney and retina $(2,9,12,14,28,51-54)$. Our previous studies demonstrated that $\mathrm{ABCG}^{+}$MPCs localize to remodeling parenchyma and microvessels following bleomycin injury and during peak fibrosis (12). Here, we expand these findings and evaluate the functional significance of abnormal lineage specification of $\mathrm{ABCG} 2^{+} \mathrm{MPCs}$ in response to injury. We performed comparative bleomycininduced fibrosis experiments using $\beta \mathrm{OE}$ and WT lineage-labeled mice (Figure 4 and Supplemental Figure 7). Following bleomycin injury, the WT MPC populations significantly increased in number on days $2-14$, while the $\beta O E$ BMC population doubled between days 7 and 14, which was quantitatively 3-fold greater than the WT MPC population on day 14 (Supplemental Figure 7). In response to bleomycin, relative to WT mice, the $\beta \mathrm{OE}$ mice had increased mortality, increased fibrosis as measured by Ashcroft scoring and collagen content, as well as increased Fulton indices, indicative 
A

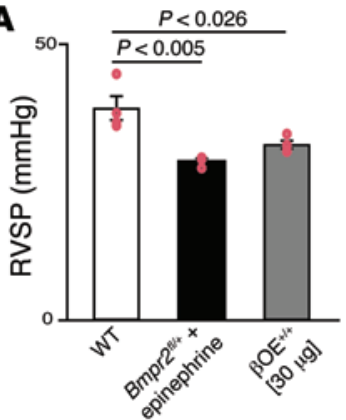

B

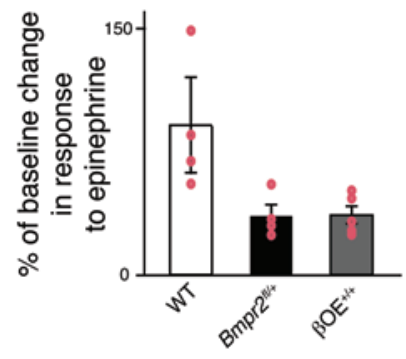

C
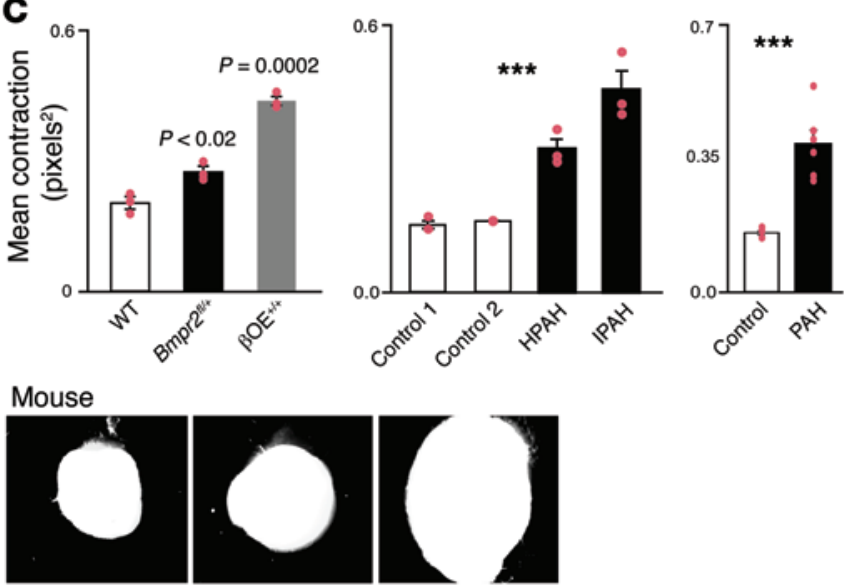

Human

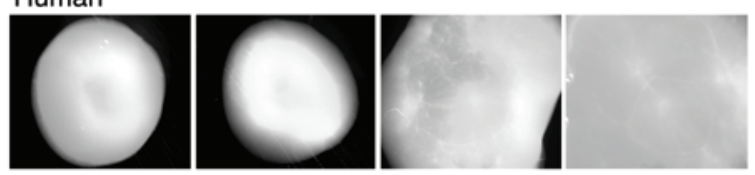

of PVD (Figure 4, A-D, F and G). Further analyses of the pulmonary microvasculature identified both decreased $\mathrm{F} 8$ - and $\alpha$-SMAexpressing microvessels, suggesting decreased microvascular density (Figure 4, E, H-K). Taken together, our data demonstrate that activation of Wnt $/ \beta$-catenin signaling in $\mathrm{ABCG} 2^{+}$MPCs exacerbates the development of fibrosis, with an underlying defect in the pulmonary microvasculature.

Wnt/ $\beta$-catenin-activated $A B C G 2^{+}$lung MPCs participate in abnormal angiogenesis during fibrosis. In order to ascertain an underlying cell-based mechanism for the exacerbated fibrosis observed, we performed lineage-tracing analysis in this murine model of bleomycin-induced fibrosis as well as analyses of isolated human idiopathic pulmonary fibrosis (IPF), MPC global gene expression profiles, and microvascular histology. In bleomycin-injured lung tissue, eGFP labeled the $\beta \mathrm{OE}$ MPC lineage that formed $\alpha$-SMA structures resembling microvessels in the distal lung (Figure 5, A, $\mathrm{C}, \mathrm{E})$. Further analysis of endothelial investment of the structures via $\mathrm{F} 8$ localization demonstrated that the vascular structures did not have an endothelium-lined lumen (Figure 5, B, D, F).

We next analyzed isolated human lung $\mathrm{ABCG}^{+}$MPCs to determine whether these findings could translate to the microvasculature in the chronic lung disease IPF. We performed global gene expression analysis to characterize the molecular signatures of IPF patient-derived ABCG2 $2^{+}$MPCs. Functional association protein networks were synthesized for genes that were differen-
Figure 3. ABCG2+ lung MPCs regulate the contractility and function of microvessels. ( $A$ and $\mathbf{B}$ ) The murine strains indicated were induced with i.p. tamoxifen ( $0.5 \mathrm{mg}$ total). Eighteen to twenty weeks after tamoxifen induction, a pressure transducer was placed into the jugular vein to the right heart to measure RVSP. Baseline pressures were measured, and epinephrine was injected i.v. into the femoral vessel $(n=5)$. $P$ values in A were determined by by 1-way ANOVA followed by Tukey's post-hoc test. (C) In vitro contractility of isolated primary murine lung MPCs and human control and PAH ABCG2 $2^{+}$MPCs was measured by plating cells onto collagen discs at time zero. The discs were photographed and the area calculated using Imagej. Shown are representative collagen gel images from 2 independent replicates. Data are presented as the mean \pm SEM. ${ }^{* * *} P<0.001$. $P$ values in $\mathbf{C}$ were determined by by 1-way ANOVA followed by Tukey's post-hoc test (for murine qRT-PCR) and a nonparametric Wilcoxon-KruskalWallis test and $\chi^{2}$ approximation (for patients' samples).

tially expressed in IPF ABCG2+ MPCs compared with control MPCs on the basis of a significant absolute increased or decreased fold change of more than $1.7(P<0.05$, by moderated $t$ test). Interestingly, both Wnt and BMPR signaling pathways were highlighted along with smooth muscle contraction, matrix, angiogenesis, and cell-cycle regulation (Supplemental Figure 8 and Supplemental Table 3). We found that IPF ABCG2 ${ }^{+}$MPCs expressed increased levels of the pericyte markers CSPG4, ACTA2, collagens (COL1A1 and COL1A3), and CCND1 (encoding cyclin D1), with decreased expression of the Wnt inhibitors DKK1 and SFRP1 (Figure 5G), similar to what was observed in PAH ABCG2 $2^{+}$MPCs (Figure 2A). Molecular profiling of IPF patient-derived lung MPCs therefore defined a link between Wnt and BMP signaling in the maintenance of the MPC phenotype.

We next examined human lung tissue samples from control, IPF, and HPAH patients for the existence of NG2-, $\alpha$-SMA-, and NG2-lined microvessels. NG2 and $\alpha$-SMA expression was colocalized in both remodeled IPF and PAH microvessels (Figure 5H). NG2-lined microvessels were also detected in IPF lung tissue in areas of fibrosis (Figure 5I). On the basis of these data, we predict that $\mathrm{ABCG} 2^{+} \mathrm{MPC}$ dysfunction, due to increased canonical Wnt signaling activity, causes abnormal microvascular function and defective angiogenesis during repair, resulting in exacerbated tissue remodeling. While it may seem counterintuitive to study a small population of MPCs as a contributor to PVD disease pathology, the innate characteristics that define them as progenitor cells make them an ideal candidate. The capacity of these MPCs to differentiate, specifically into pericytes, highlights them as an ideal cell type to influence normal and abnormal vascular function. These MPCs may contribute to the PVD associated with adult lung diseases by abnormal lineage differentiation as well as by influencing surrounding endothelial cell (EC) structures (Figure 6) (55).

\section{Discussion}

The findings from these studies expand our understanding of the role lung MPCs, and the pericytes derived from them, play during pulmonary microvascular homeostasis and adaptive angiogenesis following injury. We demonstrate that ABCG2 $2^{+}$MPCs directly influence lung microvascular function. Specifically, this work 
A
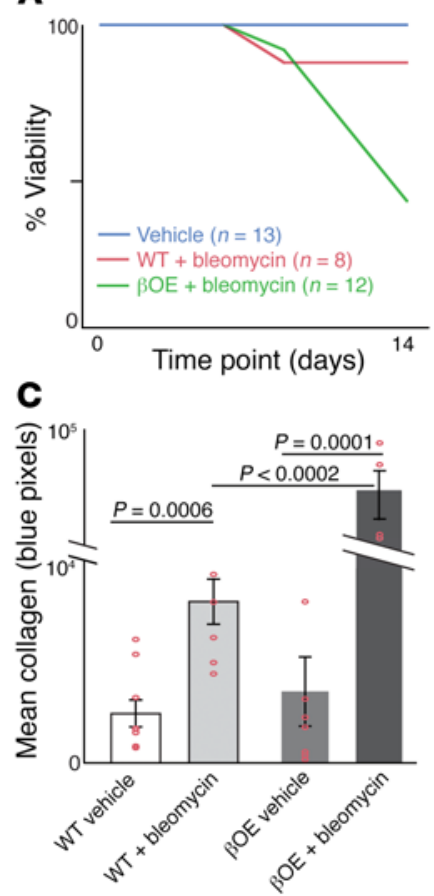

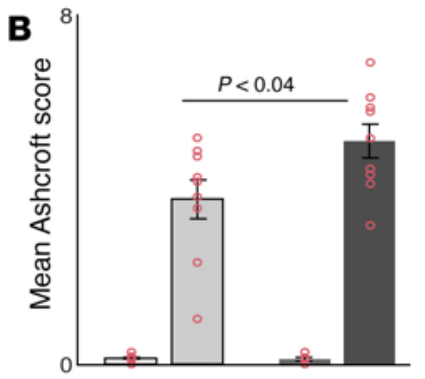

D

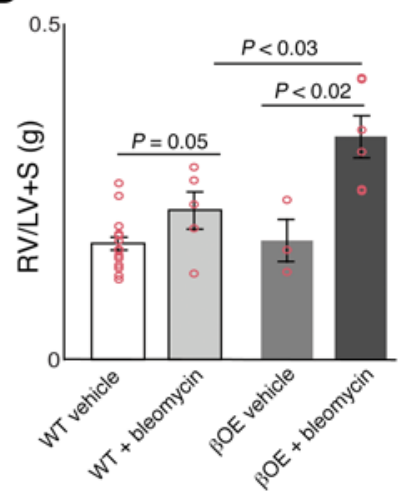

E

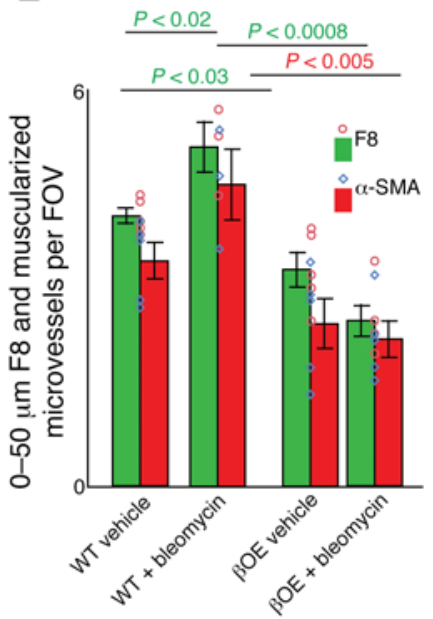

$\mathbf{F}$

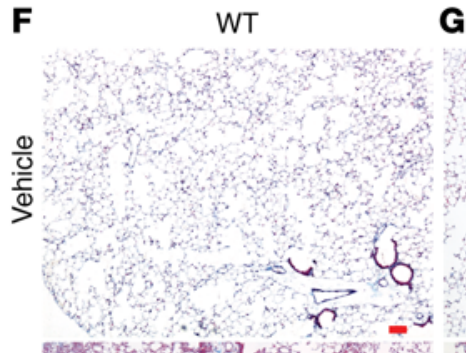

G $\quad \beta O E$
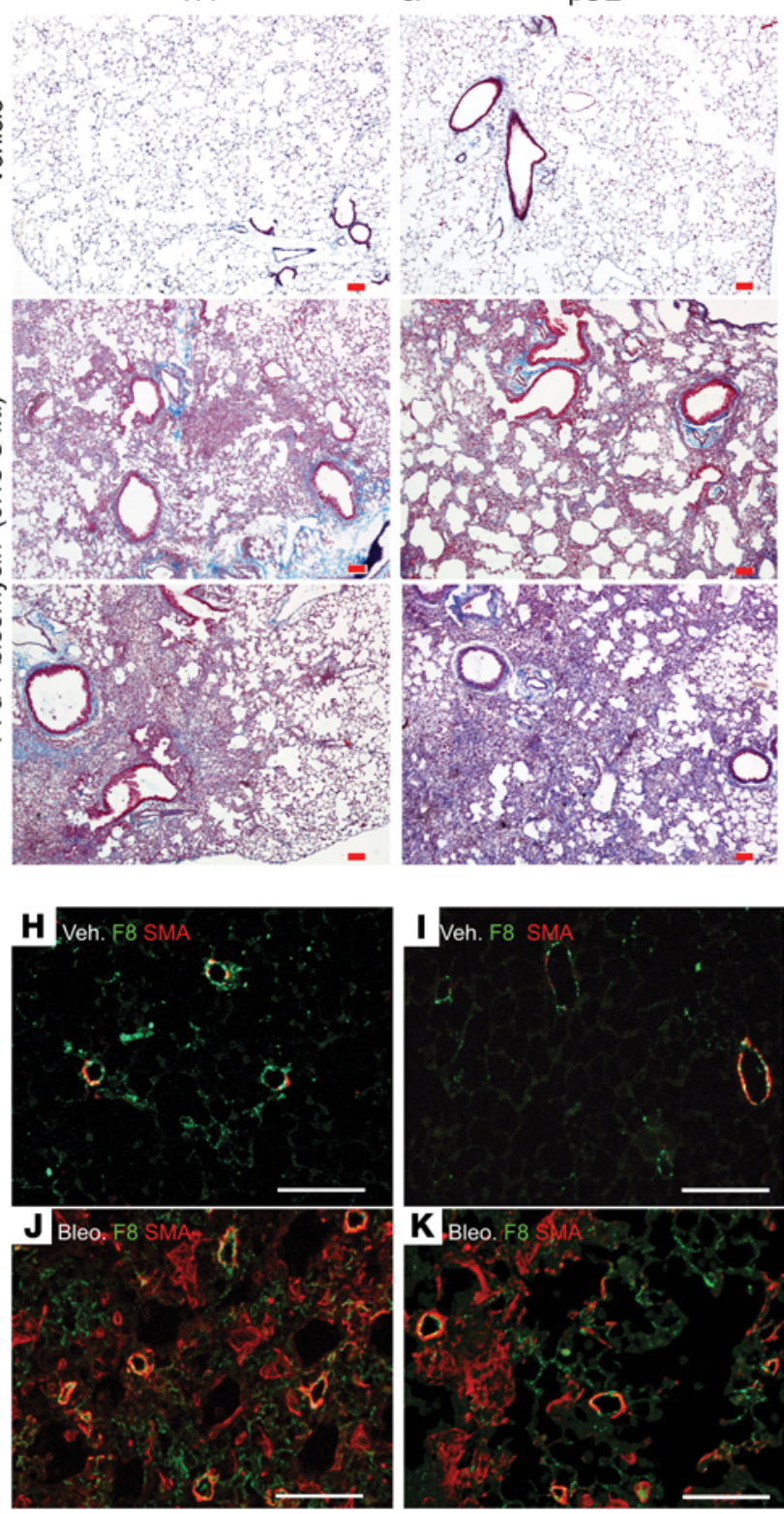

Figure 4. Disruption of lineage specification by canonical Wnt activation in ABCG2+ MPCs exacerbates bleomycin injury. Adult WT and $\beta O E$ mice were induced with i.p. tamoxifen ( $0.5 \mathrm{mg}$ total). Two weeks after induction, $0.15 \mathrm{U}$ bleomycin or PBS vehicle was administered intratracheally (i.t.), and mice were analyzed on day 14 during peak fibrosis. (A) The mean mortality was recorded. (B) Blinded Ashcroft scoring was used to quantitate fibrosis using trichrome-stained sections $(n=9$ ). (C) Collagen was quantitated using custom Fiji software and imaging of 15 FOV on 3 slides per mouse. (D) The Fulton index was calculated as an indirect measure of PVD. RV/LV+S, right ventricle/left ventricle + septum. (E) Immunostaining was performed on lung tissue sections to detect F8 $8^{+}$microvessels and $\alpha-5 \mathrm{MA}^{+}$muscularized vessels. Positive vessels of less than $50 \mu \mathrm{m}$ were counted per FOV. Four sections of twenty FOV per section per mouse were evaluated $(n=4)$. Shown are representative images of trichrome- $(\mathbf{F}$ and $\mathbf{G})$ and F8- and $\alpha$-SMA-immunostained $(\mathbf{H}-\mathbf{K})$ lung tissues. Data are presented as the mean \pm SEM. (B-E) Murine data were analyzed by 1-way ANOVA followed by Tukey's post-hoc test. Scale bars: 100 um.

delineates that the coordinated regulation of Wnt/ $\beta$-catenin signaling in $\mathrm{ABCG} 2^{+}$mesenchymal pericyte progenitors, autonomously or downstream of BMPR/TGF- $\beta$ signaling, is a key determinant of lung microvascular integrity and is intimately linked with the alveolar epithelium. Indeed, increased canonical Wnt signaling enhanced ABCG2 $2^{+}$MPC proliferation and promoted the specification of these cells to functionally deficient, proangiogenic pericytes, in lieu of their active contribution to myofibroblast accumulation with fibrosis (Figure 6). Importantly, these findings affirm that maintaining a proper balance of Wnt signaling in lung $\mathrm{ABCG} 2^{+}$MPCs promotes proper pericyte development for the maintenance of microvascular homeostasis and the regulation of angiogenesis following injury.

Pericytes may be considered the SMC of the microvessels or capillary beds $(28,56)$. While related in lineage, the pericyte is typically more intimately associated with the endothelium than 

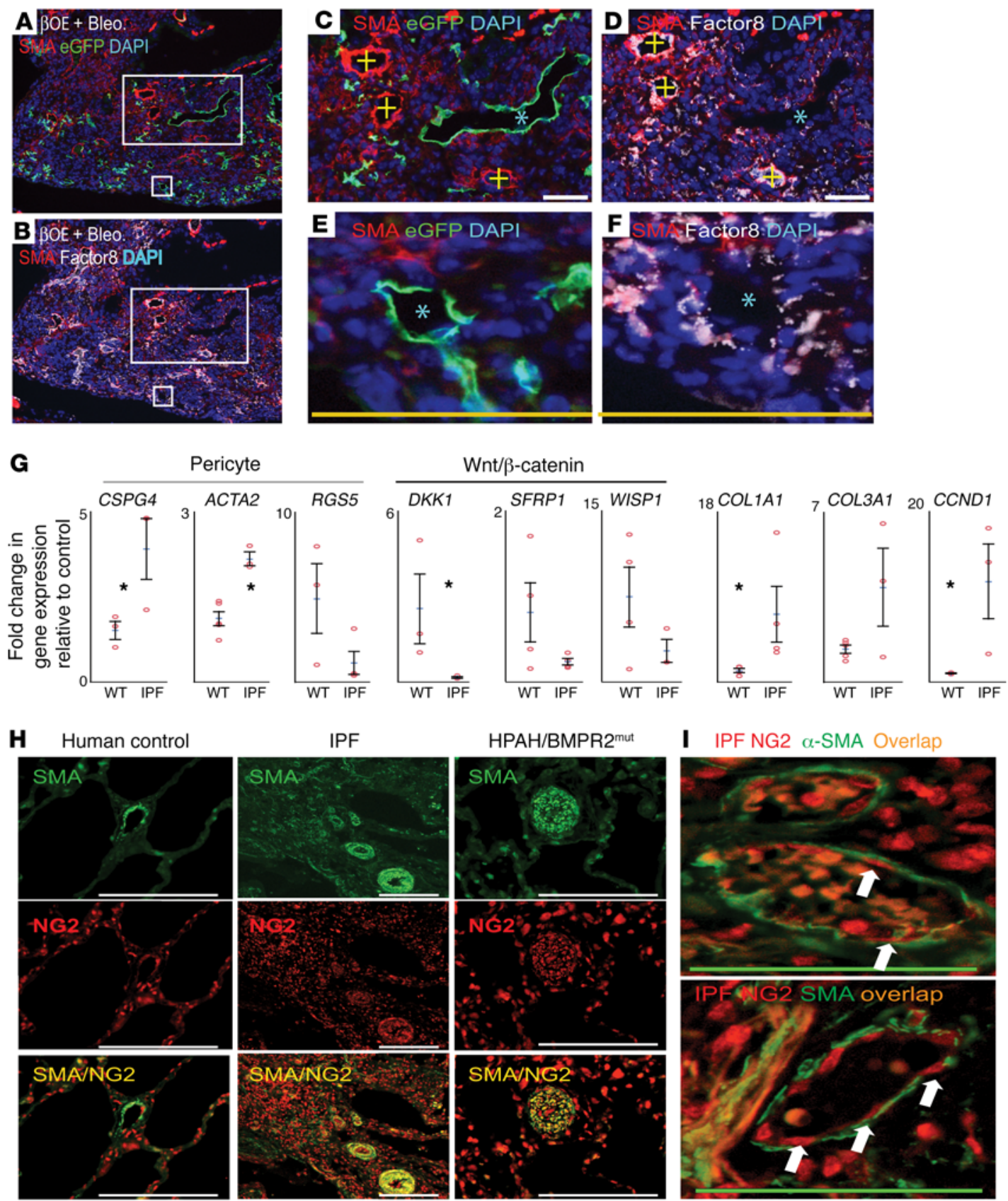

Figure 5. Wnt activation of lung MPCs promotes the formation of abnormal vascular structures during fibrosis. (A-F) Adult WT and $\beta O E$ mice were induced with i.p. tamoxifen ( $0.5 \mathrm{mg}$ total) and treated i.t. with $0.15 \mathrm{U}$ bleomycin or PBS vehicle 2 weeks later. Immunostaining was performed on sections of lineage-labeled mouse lungs on day 14 to localize eGFP-labeled (green) MPCs and their derivatives as well as $\alpha$-SMA (red) and F8 (white). Representative images from $\beta O E$ mouse lungs are shown $(n=5-6)$. (C) Human IPF ABCG2+ lung MPCs showed an increased pericyte lineage commitment and alterations in Wnt target gene expression as detected by qRT-PCR. The pericyte lineage markers CSPG4, ACTA2, and RGS5, the Wnt pathway targets DKK1, SFRP1, and WISP1, as well as the matrix proteins COL1A1 and COL1A3 and the cell-cycle regulator CCND1 were analyzed $(n=3-4) .{ }^{*} P<0.05$, by nonparametric Wilcoxon-Kruskal-Wallis test. (H) Human control, IPF, and HPAH tissue sections were immunostained to detect NG2 (red) and $\alpha$-SMA (green) $(n=3)$. Overlapping expression of $\alpha$-SMA and NG2 (yellow) was localized to microvascular regions of remodeling. (I) NG2-lined microvessels were also present in human IPF lung tissue. Arrows indicate luminal NG2+ cells. Scale bars (C-F, H, and I): $50 \mu \mathrm{m}$. Original magnification $(\mathbf{A}$ and $\mathbf{B}): \times 4$. Images in $\mathbf{C}$ and $\mathbf{D}$ are enlargements of the highlighted boxes in $\mathbf{A}$, and images in $\mathbf{D}$ and $\mathbf{F}$ are enlargements of highlighted boxes in $\mathbf{B}$.

are vSMCs, which are also distinguished by location, morphology, and markers (57). vSMC phenotypic plasticity has been described in multiorgan development as well as in various adult diseases characterized by vascular remodeling $(49,50)$. The plasticity of vSMCs is represented by 2 major phenotypes: differentiated and contractile and dedifferentiated, proliferative, migratory, and synthetic (48-50), similar to the differentiation of MPCs into the pericyte lineage we have documented in these studies. To date, the molecular mechanisms underlying the phenotypic switches and their regulation of homeostasis and adaptive versus patholog- 
Homeostasis

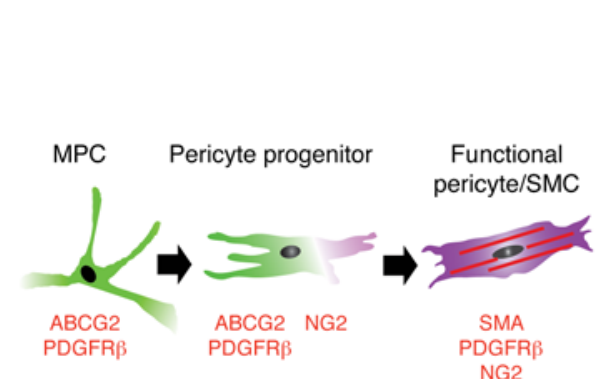

Pulmonary microvascular dysfunction

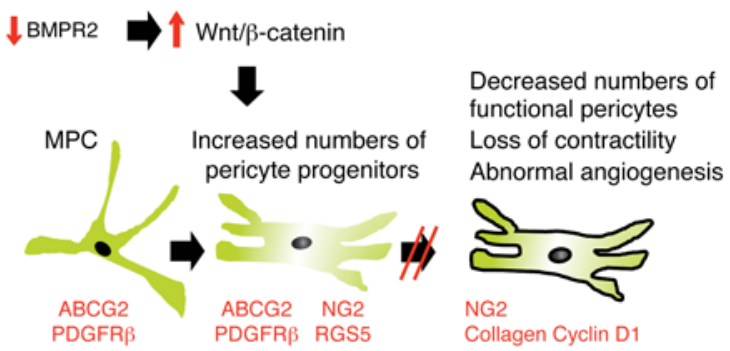

Figure 6. Schematic showing that disruption of lineage specification in adult pulmonary MPCs promotes microvascular dysfunction. Schematic summary of the presented data.

ical angiogenesis remain to be deciphered. Thus, there is a paucity of knowledge defining the process of de novo pericyte differentiation and maturation from the amorphous mesenchyme in the adult lung. Appropriate mesenchymal specification to pericytes is likely important during microvascular homeostasis, maintenance of the distal airspace structure, and development of the vascular dysfunction associated with chronic lung diseases (58).

The lack of suitable markers to trace mesenchymal-topericyte differentiation in the adult lung has limited our understanding of the role of pericytes in homeostasis and disease. Here, we established ABCG2 as a genetic marker that facilitates the study of adult pericyte progenitor specification from the pulmonary mesenchyme. While ABCG2 has been used as a marker of adult tissue stem cells, first in bone marrow hematopoietic stem cells (BM-HSCs), followed by our work defining ABCG2 ${ }^{+}$ $\mathrm{BM}-$ and skeletal muscle-derived angioblasts $(59,60)$, its use as a genetic marker for adult mesenchymal pericyte progenitors has not, to our knowledge, been previously reported. Currently, there is no lung-specific vascular cell marker to study lineage tracing in adult diseases. For example, PDGFR $\beta$ and GLI family zinc finger 1 (GLI1) have been used to trace mesenchymal cells during fibrosis and development, but these markers label mixed mesenchymal cell populations as well as epithelial cell lineages in the adult, respectively (Supplemental Table 1) (61-63). Last, current models rely on smooth muscle protein $22 \alpha$-driven (SM220driven) or SM-MHC-driven Cre systems to manipulate genes in lung smooth muscle (64-66), but SM22 and SM-MHC demonstrate systemic expression. Endothelial cell lineage tracing has been reported using Tie2-Cre (67), which labels mesenchymal cells as well as circulating BM-derived angioblasts (59). The results of these studies validate the use of $A B C G 2$ as a genetic marker to study resident mesenchymal pericyte progenitors in the adult lung during homeostasis repair and disease processes.

The validation of ABCG2 as a marker of adult mesenchymal pericyte progenitors enabled us to determine how the genetic stabilization of $\beta$-catenin in these cells or depletion of BMPR 2 affected pericyte formation and lung microvascular function. Conditional genetic stabilization of $\beta$-catenin or depletion of BMPR2 in $\mathrm{ABCG} 2^{+} \mathrm{MPCs}$ in vivo resulted in the expansion of this progenitor pool. These cells exhibited pericyte lineage specification in vitro, however, they did not assume contractile function or expression of smooth muscle actin in our in vivo or in vitro studies. Early expan- sion of the mesenchymal progenitors correlated with pulmonary vascular leak, likely as a result of decreased association of the mesenchymal progenitors with the microvascular endothelium. Vascular leak is a key indicator of vascular endothelial dysfunction and is known to precede remodeling, including both microvessel pruning and muscularization (68). Thus, our findings indicate that $\mathrm{ABCG}^{+} \mathrm{MPC}$-to-pericyte differentiation is an important determinant in the maintenance of proper lung function and health.

The impact of $\mathrm{ABCG}_{2}{ }^{+}$mesenchymal progenitors on tissue function probably increased as these progenitors accumulated over time. In fact, prolonged $\mathrm{ABCG}^{+}$stabilization of $\beta$-catenin and depletion of BMPR2 decreased terminal or functional pericyte differentiation, which was confirmed by the reduced expression of $\alpha$-SMA and the loss of $\mathrm{F}^{+}$microvessel density in vivo. The distal airspace enlargement was likely due to the loss of mesenchymal progenitor function, compounded by the loss of the supporting microvasculature. The idea of a vascular component contributing to the loss of distal lung tissue structure or emphysema/chronic obstructive pulmonary disease (COPD) has been demonstrated with the use of SUGEN5416 as a VEGF blockade (69), since VEGF is a survival factor for both lung endothelial and mesenchymal cells (70). These studies highlight the fact that intact ABCG2 mesenchymal progenitor function is necessary to maintain both microvascular and alveolar epithelial survival and lung function.

Pulmonary microvascular function is impaired in both PAH and IPF as a consequence of remodeling and collagen deposition or of fibrosis, which are associated with the microvascular structures. The etiology of early or adaptive microvascular remodeling remains unknown and is complicated by conflicting evidence of angiogenesis as reparative or pathologic in both PAH and IPF $(6-8,13,71,72)$. Contradictory data also suggest that microvessel density decreases or increases during IPF, depending on the localization and severity of fibrosis (6-8). In our murine models of bleomycin-induced fibrosis, ABCG2 $2^{+}$MPCs associated with "traditional" $\alpha-\mathrm{SMA}^{+}$or $\mathrm{F}^{+}$microvessels in alveolar tissue peripheral to the actively remodeling regions. However, in $\beta \mathrm{OE}$ lung tissue, we detected MPC contribution to atypical vascular structures in the fibrotic areas that were devoid of both F8 endothelium and $\alpha$-SMA, which may be the reason the traditional vascular density was seen to be decreased in the scar region (6-8). The finding of these structures in $\beta \mathrm{OE}$, but not WT, lung tissue may be due to the increase in labeled lineage-derived cells (3-fold more than 
in WT tissue). Additionally, the increased expression of NG2 or decreased CD105 by these cells may increase their angiogenic and invasive nature $(31,36)$. However, whether increased Wnt signaling in MPCs results in long-term fibrosis or enhanced repair is an avenue of current investigation.

In these studies, we provide the first evidence to our knowledge that Wnt/ $\beta$-catenin signaling regulates MPC-derived pericyte progenitor angiogenic function during fibrosis in the dense fibroblast "scar" region. Translating our findings in murine lungs to human disease, we identified luminal $\mathrm{NG}^{+}$microvessels in IPF lung tissue. These alternative vascular structures have been described as leading angiogenic tubes composed of pericytes during development, as well as adult tumor vasculature, where nontraditional angiogenesis was previously termed "vascular mimicry" (73-77). Abnormal vessels formed by pericytes or epithelial cells regulate cell metastases, inflammation, and the overall tumor microenvironment. In addition to influencing angiogenesis and the subsequent recruitment of endothelium, these pericytes may also influence the surrounding myofibroblasts, as pericytes in the skin have also been shown to regulate the recruitment of epithelium during wound healing in the absence of angiogenesis (78). Such mesenchyme-derived tubes could influence the extent of injury or fibrosis by influencing the tissue microenvironment.

Our current and previous studies directly link increased $\beta$-catenin signaling with deregulated BMPR signaling $(22,79)$. These data suggest that intact BMP signaling promotes the differentiation of pulmonary mesenchymal pericyte progenitors and suppresses Wnt/ $\beta$-catenin signaling, which is required for the selfrenewal and proliferation of progenitor pools. Another important observation from these studies is that conditional genetic depletion of BMPR2, stabilization of $\beta$-catenin in ABCG2 $2^{+}$MPCs, as well as bleomycin injury in vivo resulted in expansion of the progenitor pool, while terminal contractile function was not achieved. Previous studies have also reported the expansion of undifferentiated progenitor pools in other systems as a result of genetic depletion of BMPR, a decrease of BMP signaling, or an increase of $\beta$-catenin activation (80-88). Conditionally decreased BMPR signaling in adult mice drove the expansion of progenitor pools in which terminal differentiation in multiple cell types was inhibited via the activation of $\beta$-catenin signaling (80-82). $\beta$-Catenin signaling has also been identified as an essential component of stem cell, mesenchymal cell, and SMC proliferation and differentiation in multiple models of development (83-88). Therefore, the effects of Wnt/ $\beta$-catenin activation are specific to the cell type, stage of differentiation, and the factors present in the microenvironment.

Studies of adult differentiated vSMCs and pericytes have previously reported the coordinated regulation of both canonical and noncanonical Wnt signaling by BMP. Genetic mutations in the BMPR2 gene or dysregulated BMPR2 signaling are associated with the development of PVD including pulmonary veno-occlusive disease (PVOD) (89), PAH $(90,91)$, as well as PH associated with COPD and pulmonary fibrosis (PF) (92). Thus, impaired BMPR2 signaling is a unifying feature in the pathogenesis of PVD and the development of $\mathrm{PH}$. Here, we demonstrate for the first time to our knowledge that coordinated canonical Wnt/BMPR2 signaling has an impact on distal lung architecture by regulating MPC-to-pericyte differentiation and that deregulated BMPR/
Wnt signaling is present in human IPF lung MPCs. In WT SMCs, BMP signaling activates both $\beta$-catenin and noncanonical Wnt pathways, which regulate SMC proliferation and motility in response to stimuli (20). Primary pericytes derived from IPAH or WT lung explants demonstrate a decreased association with microvessels in vitro that is attributed to abnormal noncanonical Wnt signaling (93). In contrast, increased noncanonical Wnt signaling in endothelial cells increases their survival and decreases vascular pruning in vivo and in vitro (94). Canonical Wnt/ $\beta$-catenin signaling is required for SMC development and terminal differentiation, while in the absence of SMC differentiation, adventitial precursors may proliferate (85). These studies illustrated that a differentiation defect in perivascular cells influences the surrounding mesenchyme as well as fibroblast specification, further illustrating the cell-specific and complex nature of Wnt signaling during vascular homeostasis.

In summary, our studies illustrate that $\mathrm{ABCG}^{+}$lung MPCs participate in microvascular homeostasis and distal lung tissue structure via pericyte specification and differentiation. We show, in both murine and human ABCG2+ lung MPCs, that increased Wnt/ $\beta$-catenin signaling results in abnormal expansion as well as pericyte specification of MPCs without normal pericyte maturation, driving a phenotype of persistent microvascular dysfunction. Therefore, the identification of alterations in MPC signaling and function will likely lead to the identification of novel interventional strategies. Treatment at an early stage of microvascular dysfunction may be most effective at decreasing progression or reversing the disease processes, improving the outcome and survival of patients at risk for $\mathrm{PH}$. More broadly, this knowledge may be applied to the microvascular dysfunction associated with other adult epithelial tissues, including skin and kidney.

\section{Methods}

Supplemental Experimental Procedures and associated references are provided in the supplemental materials.

Genetic manipulation of murine ABCG2 $2^{+}$MPCs. Abcg2-Cre ${ }^{E R T 2}$ mice, a gift of B. Sorrentino (St. Jude Children's Research Hospital, Memphis, Tennessee, USA) (23), were crossed with a fluorescent eGFP reporter strain [(Cg)-Gt(ROSA)26Sortm4(ACTB-tdTomato,-EGFP); stock no. 007676, designated $\mathrm{mT} / \mathrm{mG}$; The Jackson Laboratory] to facilitate lineage analysis and quantitation via eGFP expression. A third gene was crossed into the mice to knock out Bmpr2 ( ${ }^{\text {floxp }} \mathrm{Bmpr} 2$ mice, obtained from the Mutant Mouse Regional Resource Center [MMRRC] [ref. 26]) or stabilize $\beta$-catenin (Catnb ${ }^{\text {loxp }}$ [ $\Delta$ ex3] mice, a gift of M.M. Taketo, Kyoto University, Kyoto, Japan) (24). To quantitate the recombination efficiency of eGFP reporter expression and recombination at the second allele as $50 \%$ as well as the impact of its expression on microvascular function in vivo, we crossed the $A b c g 2$ driver/reporter strain with a mouse containing a floxed-STOP cassette allele, regulating the expression of diphtheria toxin A $(95,96)$ (stock no. 009669; The Jackson Laboratory) (Supplemental Figure 9, A and B). Mice were injected i.p. at 8 to 10 weeks of age with a low dose $(0.5 \mathrm{mg})$ of tamoxifen (T-5648; Sigma-Aldrich) in sesame oil or with sesame oil alone (vehicle control) $(12,19)$. The mice were randomized and distributed as 3 to 5 mice per cage for studies. To demonstrate that recombination of floxed Ctnnb1 $\Delta \mathrm{ex} 3$ was detected in the $\mathrm{eGFP}^{+}$fraction, genotyping PCR analysis was performed on freshly isolated $\mathrm{CD}_{4} 5^{-} \mathrm{eGFP}^{+}$puta- 
tive MPCs and CD45-eGFP- cells 48 hours following induction. This experiment assumed that differentiated microvascular endothelial cells (MVECs) are present in the $\mathrm{GFP}^{-}$fraction, along with other lung cells. The results showed that recombination was detectable in the $\mathrm{GFP}^{+}$fraction of putative MPCs and not the GFP fraction containing MVECs and other lung cells (Supplemental Figure 9C).

To validate that resident lung MPCs were not derived from the $\mathrm{ABCG} 2^{+}$hematopoietic population, we performed BM transplantation using murine ABCG2 lineage-labeled whole BM (Supplemental Figure 10). Genetic depletion of BMPR2 and eGFP expression was confirmed by isolating eGFP-expressing $\mathrm{ABCG} 2^{+}$lung MPCs from explanted murine lungs via flow sorting, in vitro expansion, and subsequent Western blot analysis to quantitate decreased BMPR2 protein levels (Supplemental Figure 4, C and D). Significantly enhanced canonical

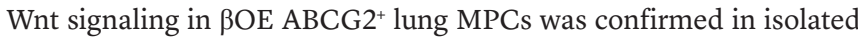
cells using a T cell factor/lymphoid-enhancer factor (TCF/LEF) luciferase reporter assay and by increased Axin 2 expression (Supplemental Figure 4, E and F).

Phenotyping of PVD. Vascular permeability in the lungs was quantitated using an AngioSense 750EX Fluorescent Imaging Agent (PerkinElmer) with spectroscopic imaging in a LI-COR Pearl Impulse Small Animal Imager (97). Elevated pulmonary artery pressure was documented indirectly by the measurement of RVSP and microvascular morphometry (98). The average interalveolar distance was assessed by quantification of MLI in lungs inflated with agarose under constant temperature and pressure as described previously $(99,100)$. Immunofluorescence staining was performed to lineage trace eGFP-labeled lung MPCs and localize $\alpha$-SMA (the reagents used are listed in Supplemental Tables 4 and 5).

Isolation and characterization of murine and human primary lung MPCs. Murine Bmpr $2^{f / /+}$ and $\beta O E$ MPCs were isolated from the lineagetracing murine strains on the basis of eGFP expression and analyzed by flow cytometry (Supplemental Figure 4A). Flow sorting, along with antibody labeling of $\mathrm{ABCG}^{+}$cells, was used to isolate human lung MPCs from lung tissue explants from WT controls as well as from a patient with a known BMPR2 mutation and decreased BMPR2 signaling; a patient with HPAH and decreased BMPR2 signaling without a known BMPR2 mutation; a patient with IPAH; and a patient with IPF. The techniques for isolation and characterization of MPCs were previously described, and ABCG2 was validated as a cell-surface marker for both murine eGFP-labeled MPCs and human MPCs (12) (Supplemental Figure 4G, Supplemental Figure 6, A-E, and Supplemental Table 2).

In vitro contraction. To test the contractility of $\mathrm{ABCG} 2^{+} \mathrm{MPCs}$ in response to treatments, cells were plated on collagen discs and photographed over time. Image (NIH) was used to calculate the area in pixels squared of each gel at each time point. The fold change in pixels squared for the 24 -hour and 48 -hour time points was calculated as the ratio of pixels squared to the gel area at the 0-hour time point.

Modulation and detection of BMPR2 and Wnt signaling. To determine the cellular response to the BMPR inhibitors dorsomorphin (DM) and dorsomorphin homolog 1 (DMH1), which were gifts of C. Hong (Vanderbilt University Medical Center), MPCs were plated at a concentration of 60,000 cells per well in medium containing $20 \%$ serum. The cells were allowed to remain in $20 \%$ serum medium for 24 hours. After 24 hours, the medium was changed to $20 \%$ serum treatment medium containing DM $(5 \mu \mathrm{M})$, DMH1 $(10 \mu \mathrm{M})$, or DMSO vehicle (22). RNA lysates were collected at 48 hours and protein lysates at 72 hours for analyses of gene and protein expression. Quantitative reverse transcription sPCR (qRT-PCR) assays were performed in triplicate (for the 3 wells collected), and the levels of analyzed genes were normalized to hypoxanthine-guanine phosphoribosyltransferase (HPRT) abundance. The primers used are presented in Supplemental Table 5. qRT-PCR experiments were repeated independently 3 times.

Transcriptome analysis. Array analysis and qRT-PCR were performed as described previously $(19,101)$, in triplicate or with 3 or more independent patient samples. Briefly, total RNA was prepared with QIAGEN RNA Isolation Kit reagents (QIAGEN) for total RNA isolation and analysis of gene expression. qRT-PCR assays were performed in triplicate, and the levels of analyzed genes were normalized to HPRT abundance (GAPDH or HPRT; the list of primers used is provided in Supplemental Table 4). Complimentary DNA generated from amplified RNA was hybridized to duplicate Affymetrix Human Gene 1.0 ST chips. Differential expression analysis was performed using Bioconductor, version 3.2 (R 3.2.2). Raw probe-level data were read using the oligo package. After normalization with RMA, a moderated $t$ test implemented in the limma package was used for differential expression testing. A minimal fold change of 1.7, up or down, and a $P$ value of less than 0.05 were used as criteria for defining differentially expressed genes. Expression values for these genes are represented in a heatmap that was generated using the heatmap function in $\mathrm{R}$. The data were deposited in the NCBI's Gene Expression Omnibus (GEO) database (GEO GSE94060). The STRING database (http://string-db. org/, accessed December 10, 2015) and PathVisio 3.2.1 (https://www. pathvisio.org/) were used to schematically represent the functional association network of differentially expressed genes.

Quantitation of collagen. Ten fields of view (FOV) per two sections, four sections per mouse, of trichrome-stained mouse lungs were photographed at $\times 20$ original magnification. The resulting color images were scanned to quantitate the number and intensity of blue (collagen) positive pixels relative to red pixels ( $n=7-8$ per group). The images were scanned using Fiji (ImageJ, version 2.0.0-rc-43/1.51a) with a custom plug-in written by M. Majka (Brentwood, Tennessee, USA). The tool was written in the ImageJ macro language (https://imagej. nih.gov/ij/developer/macro/macros.html) to compare the ratio of the blue/red color components of each pixel in a given image. This macro was designed so that all pixels that had a ratio over the desired threshold to the color white set all other pixels to the color black and returned the number of white pixels. The output was the number of pixels in the image whose blue/red ratio was above the specified threshold limit on the basis of a positive and negative control image.

Statistics. Mouse model data were analyzed by 1-way ANOVA followed by Tukey's honest significant difference (HSD) post-hoc test. Murine qPCR data were analyzed by 1-way ANOVA followed by Tukey's post-hoc test. Patients' samples were analyzed using a nonparametric Wilcoxon-Kruskal-Wallis test and $\chi^{2}$ approximation. All analyses used JMP version 5.0.12 (SAS Institute Inc.). All data are presented as the mean \pm SD or SEM. A $P$ value of less than 0.05 was considered statistically significant.

Study approval. The Vanderbilt University IACUC approved all animal procedures and protocols. These studies used banked patient cell lines obtained via IRB protocol 9401, which was approved by the IRB committee of Vanderbilt University Medical Center. Patients provided informed consent under this IRB for the generation and storage of their cell lines. 


\section{Author contributions}

Conceptualization: SMM and CG; methodology: SMM, CG, SM, EJC, NCB, RFF, WDM, EDA, ALM, ARH, JDW, and SDL; investigation: SMM, CG, SM, TSB, EJC, ARH, and JDW; writing of the original draft: SMM and DJK; review and editing of the manuscript: SMM, DJK, SDL, ARH, WDM, RFF, CG, and TSB; funding acquisition: SMM; resources: TSB, JEL, JAK, MMT, CCH, and ALM; and supervision: SMM.

\section{Acknowledgments}

The authors would like to express their appreciation to Dr. J. Fessel (Vanderbilt University Medical Center) for discussions. The authors also acknowledge the expert technical assistance provided by S. Marriott, S. Majka, Lora Hedges, S. Shay, N. Penner, and Laura Hoaglin of the University of Colorado Cancer Center (UCCC) Skin Disease Research Center (SDRC) Morphology and Phenotyping Core (P30 AR 057212); Catherine E. Alford and the members of the Research Flow Cytometry Laboratory at the Nashville VA Medical Center; and Karen Helm at the University of Colorado Cancer Center's Flow Cytometry Shared Resource (P30-CA046934 NCI). This work was funded by grants to S.M. Majka from the NIH (R01HL091105 and NIH R01HL11659701). Additional funding was also provided by the NIH (PPG-5P01HL108800-04, to JEL, principal investigator). The experiments were performed at the University of Colorado's Cancer Center Microarray core facility (NCI P30 CA 46934-14). The project was supported in part by the National Center for Research Resources (UL1 RR024975-01) and is now at the National Center for Advancing Translational Sciences (2 UL1 TR000445-06). The content is solely the responsibility of the authors and does not necessarily represent the official views of the NIH.

Address correspondence to: Susan M. Majka, Associate Professor of Medicine, Vanderbilt University, Division of Allergy, Pulmonary and Critical Care Medicine, 116121 st Avenue S, T-1218 MCN, Nashville, Tennessee 37232, USA. Phone: 303.883.8786; E-mail: Susan.M.Majka@vanderbilt.edu.
1. Beers MF, Morrisey EE. The three R's of lung health and disease: repair, remodeling, and regeneration. J Clin Invest. 2011;121(6):2065-2073.

2. Crocker DJ, Murad TM, Geer JC. Role of the pericyte in wound healing. An ultrastructural study. Exp Mol Pathol. 1970;13(1):51-65.

3. Hirschi KK, D'Amore PA. Pericytes in the microvasculature. Cardiovasc Res. 1996;32(4):687-698.

4. Crisan M, et al. A perivascular origin for mesenchymal stem cells in multiple human organs. Cell Stem Cell. 2008;3(3):301-313.

5. Guimarães-Camboa N, et al. Pericytes of multiple organs do not behave as mesenchymal stem cells in vivo. Cell Stem Cell. 2017;20(3):345-359.e5.

6. Barratt S, Millar A. Vascular remodelling in the pathogenesis of idiopathic pulmonary fibrosis. QJM. 2014;107(7):515-519.

7. Hanumegowda C, Farkas L, Kolb M. Angiogenesis in pulmonary fibrosis: too much or not enough? Chest. 2012;142(1):200-207.

8. Keane MP. Angiogenesis and pulmonary fibrosis: feast or famine? Am J Respir Crit Care Med. 2004;170(3):207-209.

9. Birbrair A, et al. Type-1 pericytes accumulate after tissue injury and produce collagen in an organ-dependent manner. Stem Cell Res Ther. 2014;5(6):122.

10. Duffield JS. The elusive source of myofibroblasts: problem solved? Nat Med. 2012;18(8):1178-1180.

11. Hung C, et al. Role of lung pericytes and resident fibroblasts in the pathogenesis of pulmonary fibrosis. Am JRespir Crit Care Med. 2013;188(7):820-830.

12. Marriott $S$, et al. ABCG2pos lung mesenchymal stem cells are a novel pericyte subpopulation that contributes to fibrotic remodeling. Am J Physiol, Cell Physiol. 2014;307(8):C684-C698.

13. Noble PW, Barkauskas CE, Jiang D. Pulmonary fibrosis: patterns and perpetrators. J Clin Invest. 2012;122(8):2756-2762.

14. Rock JR, et al. Multiple stromal populations contribute to pulmonary fibrosis without evidence for epithelial to mesenchymal transition. Proc Natl Acad Sci U S A. 2011;108(52):E1475-E1483.
15. Steinhauser ML, Lee RT. Pericyte progenitors at the crossroads between fibrosis and regeneration. Circ Res. 2013;112(2):230-232.

16. Xie T, et al. Transcription factor TBX4 regulates myofibroblast accumulation and lung fibrosis. J Clin Invest. 2016;126(8):3063-3079.

17. Austin ED, Kawut SM, Gladwin MT, Abman SH. Pulmonary hypertension: NHLBI Workshop on the Primary Prevention of Chronic Lung Diseases. Ann Am Thorac Soc. 2014;11(Suppl 3):S178-S185.

18. Cojocaru M, Cojocaru IM, Silosi I, Vrabie CD. Associated pulmonary arterial hypertension in connective tissue diseases. Maedica (Buchar). 2011;6(2):141-145.

19. Chow K, et al. Dysfunctional resident lung mesenchymal stem cells contribute to pulmonary microvascular remodeling. Pulm Circ. 2013;3(1):31-49.

20. Perez VA, et al. BMP promotes motility and represses growth of smooth muscle cells by activation of tandem Wnt pathways. J Cell Biol. 2011;192(1):171-188.

21. Diebold I, et al. BMPR2 preserves mitochondrial function and DNA during reoxygenation to promote endothelial cell survival and reverse pulmonary hypertension. Cell Metab. 2015;21(4):596-608.

22. West JD, et al. Identification of a common Wnt-associated genetic signature across multiple cell types in pulmonary arterial hypertension. Am JPhysiol, Cell Physiol. 2014;307(5):C415-C430.

23. Fatima S, Zhou S, Sorrentino BP. Abcg2 expression marks tissue-specific stem cells in multiple organs in a mouse progeny tracking model. Stem Cells. 2012;30(2):210-221.

24. Harada N, et al. Intestinal polyposis in mice with a dominant stable mutation of the beta-catenin gene. ЕMBO J. 1999;18(21):5931-5942.

25. Muzumdar MD, Tasic B, Miyamichi K, Li L, Luo L. A global double-fluorescent Cre reporter mouse. Genesis. 2007;45(9):593-605.

26. Hong KH, et al. Genetic ablation of the BMPR2 gene in pulmonary endothelium is sufficient to predispose to pulmonary arterial hypertension.
Circulation. 2008;118(7):722-730.

27. Kuznetsov SA, Mankani MH, Bianco P, Robey PG. Enumeration of the colony-forming unitsfibroblast from mouse and human bone marrow in normal and pathological conditions. Stem Cell Res. 2009;2(1):83-94.

28. Armulik A, Genové G, Betsholtz C. Pericytes: developmental, physiological, and pathological perspectives, problems, and promises. Dev Cell. 2011;21(2):193-215.

29. Simonavicius N, et al. Pericytes promote selective vessel regression to regulate vascular patterning. Blood. 2012;120(7):1516-1527.

30. Mitchell TS, Bradley J, Robinson GS, Shima DT, $\mathrm{Ng}$ YS. RGS5 expression is a quantitative measure of pericyte coverage of blood vessels. Angiogenesis. 2008;11(2):141-151.

31. Nicolosi PA, Dallatomasina A, Perris R. Theranostic impact of NG2/CSPG4 proteoglycan in cancer. Theranostics. 2015;5(5):530-544.

32. Königshoff M, et al. WNT1-inducible signaling protein-1 mediates pulmonary fibrosis in mice and is upregulated in humans with idiopathic pulmonary fibrosis. J Clin Invest. 2009;119(4):772-787.

33. Blocki A, et al. Not all MSCs can act as pericytes: functional in vitro assays to distinguish pericytes from other mesenchymal stem cells in angiogenesis. Stem Cells Dev. 2013;22(17):2347-2355.

34. Espagnolle N, Guilloton F, Deschaseaux F, Gadelorge M, Sensébé L, Bourin P. CD146 expression on mesenchymal stem cells is associated with their vascular smooth muscle commitment. J Cell Mol Med. 2014;18(1):104-114.

35. Mancini ML, et al. Endoglin is required for myogenic differentiation potential of neural crest stem cells. Dev Biol. 2007;308(2):520-533.

36. Mano Y, et al. The loss of endoglin promotes the invasion of extravillous trophoblasts. Endocrinology. 2011;152(11):4386-4394.

37. Fusté NP, et al. Cytoplasmic cyclin D1 regulates cell invasion and metastasis through the phosphorylation of paxillin. Nat Commun. 2016;7:11581.

38. Sumrejkanchanakij P, Tamamori-Adachi M, Matsu- 
naga Y, Eto K, Ikeda MA. Role of cyclin D1 cytoplasmic sequestration in the survival of postmitotic neurons. Oncogene. 2003;22(54):8723-8730.

39. Hong CC, Yu PB. Applications of small molecule BMP inhibitors in physiology and disease. Cytokine Growth Factor Rev. 2009;20(5-6):409-418.

40. Dominici $\mathrm{M}$, et al. Minimal criteria for defining multipotent mesenchymal stromal cells. The International Society for Cellular Therapy position statement. Cytotherapy. 2006;8(4):315-317.

41. Cho H, Kozasa T, Bondjers C, Betsholtz C, Kehrl JH. Pericyte-specific expression of Rgs5: implications for PDGF and EDG receptor signaling during vascular maturation. FASEB $J$. 2003;17(3):440-442.

42. Holobotovskyy V, et al. Regulator of G-protein signaling 5 controls blood pressure homeostasis and vessel wall remodeling. Circ Res. 2013;112(5):781-791.

43. Berger M, Bergers G, Arnold B, Hämmerling GJ, Ganss R. Regulator of G-protein signaling-5 induction in pericytes coincides with active vessel remodeling during neovascularization. Blood. 2005;105(3):1094-1101.

44. Ono M, Inkson CA, Kilts TM, Young MF. WISP-1/ CCN4 regulates osteogenesis by enhancing BMP-2 activity. J Bone Miner Res. 2011;26(1):193-208.

45. Meyrick B, Reid L. Pulmonary hypertension. Anatomic and physiologic correlates. Clin Chest Med. 1983;4(2):199-217.

46. Oka M, et al. Rho kinase-mediated vasoconstriction is important in severe occlusive pulmonary arterial hypertension in rats. Circ Res. 2007;100(6):923-929.

47. Carraro G, et al. miR-142-3p balances proliferation and differentiation of mesenchymal cells during lung development. Development. 2014;141(6):1272-1281.

48. Liu R, Bauer AJ, Martin KA. A new editor of smooth muscle phenotype. Circ Res. 2016;119(3):401-403.

49. Owens GK, Kumar MS, Wamhoff BR. Molecular regulation of vascular smooth muscle cell differentiation in development and disease. Physiol Rev. 2004;84(3):767-801.

50. Rensen SS, Doevendans PA, van Eys GJ. Regulation and characteristics of vascular smooth muscle cell phenotypic diversity. Neth Heart J 2007;15(3):100-108.

51. Dulmovits BM, Herman IM. Microvascular remodeling and wound healing: a role for pericytes. Int J Biochem Cell Biol. 2012;44(11):1800-1812.

52. Humphreys BD. Targeting pericyte differentiation as a strategy to modulate kidney fibrosis in diabetic nephropathy. Semin Nephrol. 2012;32(5):463-470.

53. Kida Y, Duffield JS. Pivotal role of pericytes in kidney fibrosis. Clin Exp Pharmacol Physiol. 2011;38(7):467-473.

54. Lin SL, Kisseleva T, Brenner DA, Duffield JS. Pericytes and perivascular fibroblasts are the primary source of collagen-producing cells in obstructive fibrosis of the kidney. Am J Pathol. 2008;173(6):1617-1627.

55. Valluru M, Staton CA, Reed MW, Brown NJ. Transforming growth factor- $\beta$ and endoglin signaling orchestrate wound healing. Front Physiol. 2011;2:89.
56. Baskir R, et al. Pulmonary Vascular Remodeling by Resident Lung Stem and Progenitor Cells. In: Firth A, Yuan JXJ, eds. Lung Stem Cells in the Epithelium and Vasculature. Switzerland: Springer International Publishing; 2015:221-240.

57. Armulik A, Abramsson A, Betsholtz C. Endothelial/pericyte interactions. Circ Res. 2005;97(6):512-523.

58. Kasahara Y, et al. Inhibition of VEGF receptors causes lung cell apoptosis and emphysema. JClin Invest. 2000;106(11):1311-1319.

59. Jackson KA, et al. Regeneration of ischemic cardiac muscle and vascular endothelium by adult stem cells. J Clin Invest. 2001;107(11):1395-1402.

60. Majka SM, Jackson KA, Kienstra KA, Majesky MW, Goodell MA, Hirschi KK. Distinct progenitor populations in skeletal muscle are bone marrow derived and exhibit different cell fates during vascular regeneration. JClin Invest. 2003;111(1):71-79.

61. Bonner JC. Regulation of PDGF and its receptors in fibrotic diseases. Cytokine Growth Factor Rev. 2004;15(4):255-273.

62. Kramann R, et al. Perivascular Gli1+ progenitors are key contributors to injury-induced organ fibrosis. Cell Stem Cell. 2015;16(1):51-66.

63. Li C, et al. Progenitors of secondary crest myofibroblasts are developmentally committed in early lung mesoderm. Stem Cells. 2015;33(3):999-1012.

64. West J, et al. Mice expressing BMPR2R899X transgene in smooth muscle develop pulmonary vascular lesions. Am J Physiol Lung Cell Mol Physiol. 2008;295(5):L744-L755.

65. Tada Y, et al. Molecular effects of loss of BMPR2 signaling in smooth muscle in a transgenic mouse model of PAH. Am J Physiol Lung Cell Mol Physiol. 2007;292(6):L1556-L1563.

66. Sheikh AQ, Lighthouse JK, Greif DM. Recapitulation of developing artery muscularization in pulmonary hypertension. Cell Rep. 2014;6(5):809-817.

67. Qiao L, et al. Endothelial fate mapping in mice with pulmonary hypertension. Circulation. 2014;129(6):692-703.

68. Sugita T, Hyers TM, Dauber IM, Wagner WW, McMurtry IF, Reeves JT. Lung vessel leak precedes right ventricular hypertrophy in monocrotaline-treated rats. J Appl Physiol Respir Environ Exerc Physiol. 1983;54(2):371-374.

69. Tuder RM, et al. Oxidative stress and apoptosis interact and cause emphysema due to vascular endothelial growth factor receptor blockade. $\mathrm{Am}$ J Respir Cell Mol Biol. 2003;29(1):88-97.

70. Majka S, Fox K, McGuire B, Crossno J, McGuire P, Izzo A. Pleiotropic role of VEGF-A in regulating fetal pulmonary mesenchymal cell turnover. Am J Physiol Lung Cell Mol Physiol. 2006;290(6):L1183-L1192.

71. Strieter RM, Belperio JA, Keane MP. CXC chemokines in angiogenesis related to pulmonary fibrosis. Chest. 2002;122(6 Suppl):298S-301S.

72. Tuder RM, et al. Expression of angiogenesisrelated molecules in plexiform lesions in severe pulmonary hypertension: evidence for a process of disordered angiogenesis. J Pathol. 2001;195(3):367-374.

73. Ozerdem U, Monosov E, Stallcup WB. NG2 proteoglycan expression by pericytes in pathological microvasculature. Microvasc Res. 2002;63(1):129-134.

74. Ozerdem U, Stallcup WB. Early contribution of pericytes to angiogenic sprouting and tube formation. Angiogenesis. 2003;6(3):241-249.

75. Song S, Ewald AJ, Stallcup W, Werb Z, Bergers G. PDGFRbeta+ perivascular progenitor cells in tumours regulate pericyte differentiation and vascular survival. Nat Cell Biol. 2005;7(9):870-879.

76. Carmeliet P, Jain RK. Molecular mechanisms and clinical applications of angiogenesis. Nature. 2011;473(7347):298-307.

77. Virgintino D, et al. An intimate interplay between precocious, migrating pericytes and endothelial cells governs human fetal brain angiogenesis. Angiogenesis. 2007;10(1):35-45.

78. Paquet-Fifield S, et al. A role for pericytes as microenvironmental regulators of human skin tissue regeneration. JClin Invest. 2009;119(9):2795-2806.

79. Gaskill C, et al. Shared gene expression patterns in mesenchymal progenitors derived from lung and epidermis in pulmonary arterial hypertension: identifying key pathways in pulmonary vascular disease. Pulm Circ. 2016;6(4):483-497.

80. Lee-Hoeflich ST, Causing CG, Podkowa M, Zhao $\mathrm{X}$, Wrana JL, Attisano L. Activation of LIMK1 by binding to the BMP receptor, BMPRII, regulates BMP-dependent dendritogenesis. EMBO J. 2004;23(24):4792-4801.

81. Klaus A, Saga Y, Taketo MM, Tzahor E, Birchmeier W. Distinct roles of Wnt/beta-catenin and Bmp signaling during early cardiogenesis. Proc Natl Acad Sci U S A. 2007;104(47):18531-18536.

82. Reynolds SD, et al. Conditional stabilization of beta-catenin expands the pool of lung stem cells. Stem Cells. 2008;26(5):1337-1346.

83. Cohen ED, Ihida-Stansbury K, Lu MM, Panettieri RA, Jones PL, Morrisey EE. Wnt signaling regulates smooth muscle precursor development in the mouse lung via a tenascin C/PDGFR pathway. JClin Invest. 2009;119(9):2538-2549.

84. Bohnenpoll T, Trowe MO, Wojahn I, Taketo MM, Petry M, Kispert A. Canonical Wnt signaling regulates the proliferative expansion and differentiation of fibrocytes in the murine inner ear. Dev Biol. 2014;391(1):54-65.

85. Trowe MO, et al. Canonical Wnt signaling regulates smooth muscle precursor development in the mouse ureter. Development. 2012;139(17):3099-3108.

86. Nusse R. Wnt signaling and stem cell control. Cell Res. 2008;18(5):523-527.

87. Ring A, Kim YM, Kahn M. Wnt/catenin signaling in adult stem cell physiology and disease. Stem Cell Rev. 2014;10(4):512-525.

88. De Langhe SP, et al. Formation and differentiation of multiple mesenchymal lineages during lung development is regulated by beta-catenin signaling. PLOS ONE. 2008;3(1):e1516.

89. Runo JR, et al. Pulmonary veno-occlusive disease caused by an inherited mutation in bone morphogenetic protein receptor II. Am J Respir Crit Care Med.2003;167(6):889-894.

90. Austin ED, et al. Truncating and missense BMPR2 mutations differentially affect the severity of heritable pulmonary arterial hypertension. Respir Res. 2009;10:87. 
91. Atkinson C, et al. Primary pulmonary hypertension is associated with reduced pulmonary vascular expression of type II bone morphogenetic protein receptor. Circulation. 2002;105(14):1672-1678.

92. Weitzenblum E, Chaouat A. Severe pulmonary hypertension in COPD: is it a distinct disease? Chest. 2005;127(5):1480-1482.

93. Yuan K, et al. Activation of the Wnt/planar cell polarity pathway is required for pericyte recruitment during pulmonary angiogenesis. Am J Pathol. 2015;185(1):69-84.

94. Korn C, et al. Endothelial cell-derived non-canonical Wnt ligands control vascular pruning in angiogenesis. Development. 2014;141(8):1757-1766.
95. Lee CG, et al. Studies of vascular endothelial growth factor in asthma and chronic obstructive pulmonary disease. Proc Am Thorac Soc. 2011;8(6):512-515.

96. Voehringer D, Liang HE, Locksley RM. Homeostasis and effector function of lymphopenia-induced "memory-like" $\mathrm{T}$ cells in constitutively T cell-depleted mice. J Immunol. 2008;180(7):4742-4753.

97. Bryant AJ, et al. Expression of mutant bone morphogenetic protein receptor II worsens pulmonary hypertension secondary to pulmonary fibrosis. Pulm Circ. 2015;5(4):681-690.

98. Case D, et al. Mice deficient in galectin-1 exhibit attenuated physiological responses to chronic hypoxia-induced pulmonary hypertension. Am J Physiol Lung Cell Mol Physiol. 2007;292(1):L154-L164.

99. Yoshida T, et al. Rtp801, a suppressor of mTOR signaling, is an essential mediator of cigarette smoke-induced pulmonary injury and emphysema. Nat Med. 2010;16(7):767-773.

100.Thurlbeck WM. Measurement of pulmonary emphysema. Am Rev Respir Dis. 1967;95(5):752-764.

101.Jun D, et al. The pathology of bleomycin-induced fibrosis is associated with loss of resident lung mesenchymal stem cells that regulate effector T-cell proliferation. Stem Cells. 2011;29(4):725-735. 